\title{
GERO LIETZ
}

\section{GWDA vs. Duden-Aussprachewörterbuch. Die IPA- Transkription im Phonetikunterricht}

\begin{abstract}
W nauczaniu fonetyki w polskiej germanistyce używa się dzisiaj słownika wymowy Duden-Aussprachewörterbuch (Duden 2005), ale też nadal wydanego w 1982 w Lipsku Großes Wörterbuch der deutschen Aussprache (GWDA 1982). Niniejszy artykuł ma na celu systematyczny przegląd oraz krytyczną analizę najważniejszych różnic w zakresie transkrypcji fonetycznej obu tych pozycji. Ma to umożliwić studentom i pracownikom dydaktycznym germanistyki lepsze rozpoznanie, zrozumienie oraz ocenę plusów i minusów różnych koncepcji i praktycznych rozwiązań transkrypcji IPA w języku niemieckim. Są to kwestie istotne także w odniesieniu do zapowiadanego przez wydawnictwo Walter de Gruyter na czerwiec 2009 nowego, obszernego słownika wymowy języka niemieckiego.
\end{abstract}

In der Phonetikausbildung der Germanistikstudierenden in Polen wird heute neben dem Duden-Aussprachewörterbuch (Duden 2005) auch noch häufig das 1982 in Leipzig erschienene Große Wörterbuch der deutschen Aussprache (GWDA 1982) verwendet. Ziel des Beitrages ist die systematische Darstellung und kritische Analyse wichtiger Unterschiede in der IPA-Transkription dieser Wörterbücher. Studierenden und Lehrenden soll es so erleichtert werden, die Vor- und Nachteile verschiedener Ansätze und Lösungen in der IPA-Transkription des Deutschen besser erkennen, verstehen und bewerten zu können. Das gilt auch mit Blick auf die Zukunft, denn bereits für Juni 2009 ist im Verlag Walter de Gruyter das Erscheinen eines neuen, umfangreichen Aussprachewörterbuches angekündigt.

In today's phonetic instruction at German departments in Poland there are mainly two pronunciation dictionaries used: the Duden-Aussprachewörterbuch (Duden 2005) and the still popular Großes Wörterbuch der deutschen Aussprache (GWDA 1982) published in Leipzig in 1982. The aim of this paper is to give a systematic survey and a critical analysis of the main differences occurring in the IPA phonetic transcription of the two dictionaries in question. This is to help both students and teachers in better 


\section{Gero Lietz}

identifying, understanding and assessing the strong and weak points in several approaches and solutions of German IPA transcription. Discussing these questions might be also useful with regard to the near future: in June 2009 a completely new comprehensive German pronunciation dictionary is to be published by Walter de Gruyter.

Für die Phonetikausbildung im Unterricht Deutsch als Fremdsprache, insbesondere in der Auslandsgermanistik, ist ein Aussprachewörterbuch unerlässlich. Zwar enthalten auch andere Wörterbücher, wie z. B. Rechtschreibwörterbücher, verschiedene DaF-Wörterbücher oder zweisprachige Wörterbücher bestimmte Angaben zur Aussprache, diese sind jedoch zum Teil lückenhaft, unsystematisch und sparen bestimmte problematische Phänomene aus (HIRSCHFELD / STOCK 2007:15). Hirschfeld / Stock verweisen u. a. auf das de-GruyterWörterbuch Deutsch als Fremdsprache, das von TERNES (2002:134) hinsichtlich der phonetischen Angaben als „Blamage für die deutschsprachige Lexikographie" bezeichnet wird. Auch in DaF-Lehrwerken entsprechen phonetische Übungen und die dazu gegebenen Lehrerinformationen nicht immer den Anforderungen an terminologische Korrektheit, es lassen sich eine gewisse Simplifizierung und Oberflächlichkeit bei der Behandlung phonetischer Fragen feststellen (u. a. LIETZ 2007).

Der Hauptvorzug eines speziellen Aussprachewörterbuches ist seine Systematik. Neben einer detaillierten Darstellung des gesamten Phoneminventars, der Laut-Buchstaben-Beziehungen, der Akzentuierungsregeln und der intonatorischen Besonderheiten enthält ein Aussprachewörterbuch vor allem die vollständige Transkription aller verzeichneten Wörter und Eigennamen, wozu natürlich auch Namen und Wörter fremder Herkunft zählen, deren Aussprache bestimmten Eindeutschungsregeln unterliegen kann. Eine Germanistikausbildung ausländischer Studierender ist somit ohne ein Nachschlagewerk dieser Art nicht denkbar. Ein Aussprachewörterbuch sollte obligatorischer Bestandteil der Bibliothek aller Lehrenden und Lernenden in der Auslandsgermanistik sein.

Bis in die 30er Jahre des 20. Jhd.s hinein war die von THEODOR SIEBS erstmals 1898 herausgegebene Deutsche Bühnenaussprache bestimmend für die Kodifizierung der deutschen Aussprachenorm, und zwar nicht nur im Bereich Schauspiel und Kunstgesang, sondern auch im Bereich des Deutschen als Fremdsprache. Die Hauptkritikpunkte an Siebs' Werk, das allein bis 1930 insgesamt 15 Auflagen erlebte, fassen HIRSCHFELD / STOCK (2007:5) zusammen: „Die durch Akzentuierung, Lautumgebung und Position verursachte Dynamik der Phonemrealisation mit ihren Assimilationen, Reduktionen und Elisionen blieb unberücksichtigt." Siebs forderte beispielsweise die Aspiration jedes einzelnen 
Fortisplosivs, was die Kodifizierung einer „extrem überartikulierte[n] und unnatürliche[n] Aussprache“ bedeutete (HIRSCHFELD / STOCK 2007:5). ${ }^{1}$ Auf die Entwicklung nach dem Zweiten Weltkrieg hatte auch die deutsche Teilung einen gewissen Einfluss. So wurden in den 50er Jahren Anregungen ostdeutscher Wissenschaftler für eine gründliche Überarbeitung des Siebs zunächst nicht akzeptiert (HIRSCHFELD / STOCK 2007:5); der Siebs erschien 1958 in fast unverändeter Form (DE BOOR / DIELS 1958). ${ }^{2}$ Anfang der 60er Jahre gab es sowohl in der DDR als auch in Westdeutschland Bestrebungen nach einer Neukodifizierung der deutschen Aussprache. Erstes Ergebnis war das Wörterbuch der deutschen Aussprache (WDA), das 1964 im Verlag VEB Bibliographisches Institut in Leipzig erschien. ${ }^{3}$ Die letzte Auflage dieses Wörterbuches stammt aus dem Jahre 1982, damals erschienen als Neubearbeitung und unter dem neuen Titel Großes Wörterbuch der deutschen Aussprache (GWDA). Die westdeutschen Bemühungen um eine Neukodifizierung gingen vor allem von der Dudenredaktion aus. Die Einleitung zu dem 1962 als Band 6 der

1 Zur Kritik an Siebs vgl. u. a. LotZmann (1967:234), der feststellt, ,daß die SiebsNorm weder im Schulbereich noch im öffentlichen Leben überzeugende Wirksamkeit gezeigt hat“, was vor allem daran gelegen habe, dass sich ,zwischen Norm und Realisation, zwischen Sprechideal und Sprechwirklichkeit eine fast unüberbrückbare Kluft" aufgetan habe. Auch MeINHOLD (1973:61) merkt an, dass Siebs „,nicht Bühnenlautung schlechthin kodifizierte, sondern eben nur eine bestimmte Stufe der Bühnenlautung“, die man als „Höchstlautung“ charakterisieren müsse, eine „Formstufe, die im heutigen Deutsch nur noch in der Aussprache des Gesangs erscheint, und nicht einmal hier stets konsequent" (MEINHOLD 1973:61). Durch die Vernachlässigung der Sprechrealität zugunsten dieser Höchstlautung habe Siebs ,die Kodifikation der tatsächlichen Aussprache der deutschen Hochlautung auf Jahrzehnte hinaus [...] blockiert“ (MEINHOLD 1973:59).

2 Zur Geschichte der Aussprachewörterbücher in Deutschland (von den Anfängen bis in die Gegenwart) vgl. HiRSCHFELD / STOCK (2007:3-10). Hervorzuheben sind die Ausführungen über den Marburger Professor Wilhelm Viëtor, der in seinen Untersuchungen bereits Ende des 19. Jhd.s bestimmte Ergebnisse späterer phonetischer Forschung vorwegnahm und mit seiner Ausspracheregelung zugleich ein Gegengewicht zu Siebs' Bühnenaussprache darstellte. Siebs' Wörterbuch fand allerdings in der ersten Hälfte des 20. Jhd.s eine größere Verbreitung und Wirkung. - Zum Wirken W. Viëtors vgl. auch HAKKARAINEN (1995:18f.).

3 Eine westdeutsche Würdigung des WDA (1964), besonders unter dem Aspekt der Gegenüberstellung von Norm und Realisation, findet sich bei LOTZMANN (1967: 236-238). Lotzmann vergleicht in dem Beitrag das WDA mit der damals aktuellen Auflage des Siebs (18. Auflage von 1961) und dem Duden-Aussprachewörterbuch (Duden 1962), allerdings ohne im Detail auf Unterschiede in der phonetischen Transkription einzugehen, wie es das Anliegen des vorliegenden Beitrages ist. 


\section{Gero Lietz}

Duden-Reihe herausgegebenen Aussprachewörterbuch (Duden 1962) enthielt bereits Kommentare zu einer ,gemäßigten Hochlautung“, die einigen Vorgaben der Siebsschen Bühnenaussprache widersprachen, allerdings noch keinen Niederschlag im Wörterverzeichnis selbst fanden (LOTZMANN 1967:236). Ab der 2. Auflage (Duden 1974) ist auch das Wörterverzeichnis des Duden-Aussprachewörterbuches nach der 1962 noch ,gemäßigte Hochlautung“ genannten Norm ausgerichtet, nun bezeichnet als Standardaussprache bzw. Standardlautung (HIRSCHFELD / STOCK 2007:6f.). Die Diskussionen um die Neukodifizierung gingen auch an dem Siebs-Wörterbuch nicht spurlos vorüber, das in seiner 19. Auflage (1969) - offenbar beeinflusst durch die Herausgabe des WDA in der DDR und die Kommentare zur ,gemäßigten Hochlautung“ im Duden (1962) - eine Unterscheidung zwischen „reiner“ und ,gemäßigter Hochlautung“ vornahm. Durch die Einbeziehung der „gemäßigten Hochlautung“ wurde der Siebs zwar verbessert und der Sprechrealität angenähert, allerdings zog auch diese 19. Auflage aufgrund etlicher ,phonetisch inakzeptable[r] Regelungen“" harsche Kritik auf sich (HIRSCHFELD / STOCK 2007:6). Erst im Jahre 2000 erschien ein unveränderter Nachdruck der Auflage von 1969 (DE BOOR / MOSER / WINKLER 2000).

Prinzipiell liegen also für das Deutsche drei Aussprachewörterbücher vor: der Siebs, das GWDA und der Duden. Ausgehend jedoch davon, dass die 19. Auflage des Siebs aus dem Jahre 1969 (DE BOOR / MOSER / WINKLER 2000) „als Nachschlagewerk nicht aktuell“ (HIRSCHFELD / STOCK 2007:10) ist ${ }^{4}$ und dass das GWDA (1982) nach der politischen Wende von 1989/1990 nicht wieder aufgelegt wurde und seit langem vergriffen ist, kommt man $\mathrm{zu}$ dem Schluss, dass für die Standardaussprache in der Bundesrepublik Deutschland des Jahres 2008 im Grunde nur ein verfügbares, aktuelles und akzeptables Aussprachewörterbuch existiert, und das ist der Aussprache-Duden (inzwischen in der 6., überarbeiteten und aktualisierten Auflage, Mannheim 2005). ${ }^{5}$ In dem im Weiteren vorzunehmenden Vergleich soll dem Aussprache-Duden (Duden 2005) das GWDA (1982) gegenübergestellt werden. Das GWDA (1982) mit seinem Vorläufer WDA (1964) hat die polnische Germanistik in entscheidendem Maße geprägt, was bis heute nicht nur in polnischen Publikationen zur deutschen Phonetik, sondern auch in den Bibliotheksbeständen germanistischer Institute in Polen sichtbaren Ausdruck findet. Trotz seiner

4 Eine Neuauflage des Siebs ist nach Auskunft des Verlags Walter de Gruyter (April 2008) nicht vorgesehen.

$5 \quad$ Zu dem für Juni 2009 bei de Gruyter angekündigten neuen Aussprachewörterbuch vgl. die Ausführungen am Ende dieses Beitrages. 
allgemeinen Verfügbarkeit im Buchhandel ist der Aussprache-Duden nicht in jeder germanistischen Institutsbibliothek in Polen vorhanden, zumindest nicht in der aktuellen Auflage. Andererseits kann in derselben Bibliothek das seit zwanzig Jahren vergriffene GWDA in Klassensatzstärke vertreten sein. ${ }^{6}$ Ein großer Vorteil beider hier zu vergleichender Aussprachewörterbücher - des GWDA und des Aussprache-Dudens - ist die Benutzung der internationalen Lautschrift nach dem IPA-Alphabet. ${ }^{7}$ Allerdings unterscheiden sich die Transkriptionsmodelle im GWDA und im Duden trotz dieser gemeinsamen Grundlage hinsichtlich bestimmter Details. Neben Unterschieden, die lediglich graphischer Natur sind und somit die Substanz der phonetischen Interpretation nicht tangieren, gibt es eine Reihe von Diskrepanzen, die über das rein Graphische hinausgehen und abweichende Modelle der Beschreibung der phonetischen Wirklichkeit verraten. Dass es nicht nur in der Grammatik, sondern auch in der Phonetik verschiedene Beschreibungsmodelle gibt, die in abweichenden Konventionen im Rahmen der IPA-Transkription zum Ausdruck kommen, ist

6 Ausgeklammert aus dem Vergleich bleibt der Siebs, da er zum einen kein aktuelles Nachschlagewerk darstellt (s.o.) und da er für die hier besonders interessierende Phonetik-Ausbildung polnischer Germanist(inn)en in den letzten Jahrzehnten kaum eine nennenswerte Rolle gespielt hat. Bestimmend sind stets das WDA/GWDA und der Aussprache-Duden gewesen.

Abzulehnen sind sog. ,,volkstümliche Transkriptionen“, mit der „Ausspracheprobleme vorprogrammiert [werden] “ und ,,der fremde Akzent regelrecht provoziert [wird]“, vgl. Dieling / HiRSCHFELD (2000:37), die u. a. Transkriptionsbeispiele für polnische Deutschlerner wie [zecen], [sznu:r] anführen, die in keiner Weise dazu geeignet sind, den Lernenden elementare Ausspracheregeln des Deutschen nahezubringen. Obwohl die Darstellung zielsprachlicher Laute mit den (zudem auch nicht immer eindeutigen) Buchstabenwerten der Muttersprache didaktisch mehr als fragwürdig ist, sind solche Transkriptionen bis heute immer wieder in Veröffentlichungen verschiedener Verlage anzutreffen, auch für das Polnische als Fremdsprache, vgl. KEHR (2002), wo z. B. w schronisku młodzieżowym umschrieben wird als /fßchronIsku muodscheschOwym/ (17) und $z$ przyjemnościq als /BpschyjemnOschtchong/ (24). Beispiele finden sich vor allem in Touristensprachführern, vgl. u.a. für das Polnische Sätze wie [tschi moschä mi pan/panji sräpärowatsch tä ockulari] für Czy może mi pan/pani zreperować te okulary? oder [chtschaubim/chtschauabim jakonsch uadnon pamjonntkän/jakisch uadni präsänt] für Chciałbym/chciałabym jakaś tadna pamiatkeljakiś tadny prezent (MARCOPOLO 2005:62f.). Auch für das Englische, dessen IPA-Transkription heute bereits deutschen Grundschülern zumindest passiv vermittelt wird, gibt es verblüffende Beispiele auf dem Sprachführer-Markt, vgl. Umschriften wie „ßträindsch“ (strange), „mei kämmerah wohs ßtoulèn“ (my camera was stolen) oder „ei wohs èttäckd“ (I was attacked) (WERNER-ULRICH 2006:76, 107). 
normal und grundsätzlich positiv zu bewerten. Durch die Konfrontation ausländischer Germanistikstudierender mit beiden IPA-Konventionen (GWDA und Duden) kann der Blick der Studierenden für verschiedene Interpretationsmodelle geschärft und damit zur Reflexion über die Sprechrealität des Deutschen angeregt werden. Bei der Vermittlung der IPA-Transkription in der Phonetik-Ausbildung ausländischer Germanistikstudierender stößt man jedoch immer wieder auf Probleme. Das Hauptproblem ist das der Verwirrung. Bei den Studierenden besteht der verständliche Wunsch nach Eindeutigkeit in der phonetischen Transkription. Die Frage, ob nach GWDA oder nach Duden transkribiert werden soll, kann der/die Lehrende zwar zugunsten des DudenAussprachewörterbuches entscheiden (schon allein deshalb, weil der Duden das einzige gegenwärtig allgemein verfügbare und aktuelle Aussprachewörterbuch ist), die Studierenden werden jedoch in vielen, auch neueren Lehrbüchern mit einer Transkription konfrontiert, die eher an das GWDA erinnert bzw. eine Mischung beider Konventionen darstellt. Selbst Letzteres wäre nicht weiter dramatisch, wenn die Autor(inn)en sich die Mühe machten, ihre Entscheidungen zu begründen, was jedoch nicht immer der Fall ist. Eine systematische Zusammenstellung der wichtigsten Unterschiede der IPA-Transkription in Duden und GWDA, ein Abwägen der Vor- und Nachteile beider Lautschriftkonventionen sucht man vergebens. Ein weiteres Problem sind Widersprüche innerhalb ein und desselben Aussprachewörterbuches. Denn es mindert selbstverständlich nicht den Grad der Verwirrung, wenn beispielsweise in der Einführung des Duden oder des GWDA Regularitäten beschrieben werden, die sich dann im Wörterbuchteil nicht systematisch wiederfinden lassen.

Im Folgenden sollen die wichtigsten Unterschiede in der IPA-Transkription, wie sie im Duden und im GWDA verwendet wird, dargestellt und kommentiert werden. Dabei geht es nicht um graphische Unterschiede. Denn es ist unerheblich, ob etwa der Bogen zur Kennzeichnung des unsilbischen Charakters eines Vokals, z.B. in dem Wort Linguist, wie im Duden unter dem entsprechenden Vokal [lın'gurst] oder wie im GWDA darüber erscheint. Wesentlich sind in erster Linie die Unterschiede, die auf divergierende Interpretationsmodelle schließen lassen. In diesem Zusammenhang sind vor allem folgende Bereiche interessant: Qualität und Quantität der Vokale, Diphthonge, vokalische/konsonantische Realisierung des $r$, Affrikaten, Reduktionserscheinungen (u. a. Elision des Schwa-Lautes, Assimilation in der Endsilbe -en), Assimilationen an der Wort- und Silbengrenze (Stimmtonverlust), Neueinsatz von Vokalen. Der Diskussion der Transkriptionsunterschiede schließt sich im Anhang zu diesem Beitrag eine tabellarische Übersicht an. 
GWDA vs. Duden-Aussprachewörterbuch

\section{Qualität der $a$-Laute}

Im Gegensatz zum Duden, der die $a$-Laute nur hinsichtlich ihrer Quantität unterscheidet (langes $a$ [a: , kurzes $a$ [a] bei gleicher Qualität), unterscheidet das GWDA zwischen zwei $a$-Qualitäten; einem ,helleren“ [a] und einem „dunkleren“ [a] (vgl. GWDA 1982:21). Zur Begründung wird u. a. ausgeführt: „Bei $a$ ist der Unterschied [zwischen offener und geschlossener Qualität G.L.] am geringsten und kann bei Vokalkürze praktisch vernachlässigt werden; es wird deshalb nur von einem dunkleren und einem helleren $a$ gesprochen." (GWDA 1982:27)

Die Autor(inn)en des GWDA scheinen sich des unsicheren Charakters dieser Unterscheidung durchaus bewusst gewesen zu sein, denn zwei Seiten später rechnen sie eben das „dunklere“ [a] zu den geschlossenen, das „hellere“ [a] hingegen zu den offenen Lauten, ,der Einfachheit halber“ (GWDA 1982:29), wie es heißt. Ob aber eine Gleichsetzung der $a$-Laute mit den anderen, in der Tat paarweise auftretenden Vokalphonemen (mit den bekannten Oppositionen gespannt/ungespannt, geschlossen/offen) wirklich der Einfachheit dient, muss gefragt werden, vgl. dazu die folgenden Ausführungen von MEINHOLD / STOCK (1982:89):

Dem Kurzvokal wird in den Phonetiken der deutschen Sprache zumeist eine „helle“ Klangqualität, dem langen /a:/ dagegen eine ,dunkle(re)“ zugeordnet, doch treffen diese Unterschiede für das Deutsche in so geringem Maße zu, daß eine qualitative Trennung perzeptiv kaum möglich ist. Insofern wirkt auch hier die Quantität distinktiv (Schall-Schal).

An anderer Stelle betonen MEINHOLD / STOCK (1982:82), dass sich die $a$-Phoneme ,hinsichtlich ihrer Qualität bzw. ihrer Hebungsrichtung oder ihres Gespanntheitsgrades nur unbedeutend voneinander unterscheiden“. Somit sei die Quantitätsopposition für die $a$-Phoneme untereinander ,unbestreitbar dominierend“. TERNES (1999:93) führt aus:

Jede Art von $a$ (vorderes [a], hinteres [a] und alle Zwischenwerte) ist maximal offen. Eine offenere und eine geschlossenere a-Variante kann es also nicht geben. [...] In der Literatur wird mitunter von offenem oder geschlossenem $a$ gesprochen. Diese Ausdrucksweise ist wohl in Analogie zu den anderen Paarungen entstanden. Sie ist nichtsdestoweniger falsch. ${ }^{8}$

8 Auch etliche weitere Autoren unterstreichen, dass es „kein[en] qualitativ[en] Unterschied zwischen dem kurzen und dem langen a-Vokal" gibt (HAKKARAINEN 1995:28), dass „,ür /a: a/ [...] der Unterschied eindeutig quantitativer Art [ist]“ 


\section{Gero Lietz}

Aus eigener Lehrerfahrung kann berichtet werden, dass eine Befolgung der GWDA-Kodifikation ,helleres/dunkleres $a^{\text {“ }}$ inbesondere beim langen $a$-Laut zu einer in der deutschen Standardaussprache nicht zulässigen Klangveränderung in Richtung $o$-Laute führen kann, vor der u. a. LANGHOFF (1996:52) zu Recht warnt. Man denke hier z. B. an norwegische oder besonders schwedische Deutschlernende, die aus ihren Muttersprachen in der Tat dunklere $a$-Laute mitbringen und durch die GWDA-Regelung und die daraus resultierende Lautschrift förmlich zum Übertragen ihrer muttersprachlichen Lautung auf das Deutsche verleitet werden. ${ }^{9}$ Ähnlich wie im Schwedischen gibt es auch im Niederdeutschen einen in Richtung offenes $o$ tendierenden, langen, dunklen $a$ Laut, was sich sogar in der Orthographie des Niederdeutschen niederschlagen kann, vgl. die Entsprechungen für stehen, fassen, lassen, Straße bei HERRMANN-WINTER (1999): ståhn, fåten, låten, Stråt im Gegensatz zu dem helleren $a$ in Wörtern wie dat (das), af (ab), Hart (Herz), mall (verrückt). Die mögliche Unterscheidung zwischen einem hellen und einem dunklen $a$ in manchen Mundarten ist jedoch nicht maßgebend für die Kodifizierung der Standardaussprache des Deutschen. ${ }^{10}$ In der Frage der $a$-Laute erweist sich die im

(KOHLER 1995:170). Für die Transkription hat das die Verwendung nur eines Symbols zur Folge, unterschieden nur durch die Längenbezeichnung: [a] / [a:].

Vgl. u. a. die ausführlichen kontrastiven Hinweise zu den deutschen und norwegischen $a$-Lauten bei HøYEM / ZicKFELDT (1992:34f.). Folgende didaktische Schlussfolgerung wird gezogen: „Der norwegische Lerner sollte sich daher um einen relativ, hellen' A-Laut im Deutschen bemühen, weil er dadurch eine von norwegischer Aussprachegewohnheit geprägte Lautung vermeidet und auch nicht einer von deutschen Dialekten beeinflußten Aussprache verdächtigt werden kann.“ In der Transkription, deren didaktische Bedeutung für den Phonetikunterricht nicht unterschätzt werden sollte, stellen HøYEM / ZICKFELDT $(1992: 23,35)$ folgerichtig die deutschen $a$-Laute mit dem Symbol [a / a:] , die norwegischen (dunkleren) $a$-Laute hingegen mit dem Symbol [a / a:] dar, um einerseits die Sonderstellung der $a$-Laute im deutschen Vokalsystem (keine Opposition gespannt/ ungespannt) anzuzeigen und andererseits zugleich die Unterschiede in der Klangqualität zwischen dem norwegischen und dem deutschen $a$ sichtbar zu machen.

10 Auch TERNES (1999:93) stellt regional mögliche Unterscheidungen zwischen [a] und [a] nicht in Abrede, die dann in der Tat zu Realisierungen wie [ban] vs. [ba:n] (Bann vs. Bahn) führen können, so etwa in der Regionalaussprache von Hamburg oder im Sächsischen. „Für die Standardsprache gilt diese Aussprache jedoch nicht. Daher kann sie keine Grundlage für die Analyse des Standarddeutschen abgeben." 
Aussprache-Duden sichtbare Auffassung, wonach es nur eine $a$-Qualität gibt ${ }^{11}$, als plausibler und didaktisch klüger. Sowohl für das lange [a: als auch für das kurze [a] ist ein identischer „heller Klang“ (LANGHOFF 1996:52) anzustreben. In Lehr- und Übungsbüchern der deutschen Phonetik, die für die Germanistik in Polen interessant sind, wird das Phänomen $a$-Laute verschieden gehandhabt. Zwei $a$-Qualitäten werden unterschieden bei MORCINIEC (1990:35), RAUSCH / RAUSCH (1998:290-293), MORCINIEC / PRĘDOTA (2005:106f., 125f.) und MiKoŁAJCZYK (2005:59). Von nur einer $a$-Qualität gehen u.a. DIELING / HIRSCHFELD (2000:38), STOCK / HiRSCHFELD (1996:192), HIRSCHFELD / REINKE / STOCK (2007:41, 161f.) und auch DOMIŃCZAK (1993:92-96) aus, der sich als einer der wenigen polnischen Lehrbuchautoren in der Transkription stark an den Duden anlehnt. ${ }^{12}$

\section{Diphthonge}

Eng mit der Vokalqualität verbunden ist auch die Frage der deutschen Diphthonge. Zudem ist in Bezug auf die Diphthonge auch die monophonematische oder biphonematische Deutung immer wieder diskutiert worden - eine in erster Linie theoretische Frage, die hier nicht gelöst werden soll, deren Relevanz für den Phonetik- und Transkriptionsunterricht jedoch nicht zu bestreiten ist. Im Folgenden geht es vor allem um die in der Wortgruppe mein neues Haus enthaltenen „Hauptdiphthonge des Deutschen“ (VATER 2005:9). ${ }^{13}$ In den

11 Dass diese Auffassung nicht unumstritten ist, zeigt bereits WÄNGLER (1983:105), der sich vehement gegen eine Unterscheidung der $a$-Laute ausschließlich über die Quantität ausspricht, zugleich aber auch einräumt, dass ,die rein qualitativen Unterschiede zwischen den beiden $a$-Lauten zweifellos nicht so groß sind wie zwischen anderen kurzen und langen Vokalen" (WÄNGLER 1982:108). Ein Blick in Band 4 der Duden-Reihe (Duden-Grammatik) erbringt einen ähnlichen Befund: Die deutschen $a$-Laute werden in die Opposition gespannt/ungespannt einbezogen und zudem an verschiedenen Stellen im Vokalviereck positioniert (EISENBERG 1998:31, 25). Aber auch hier klingen Zweifel an, ob diese Auffassung mit der artikulatorischen Wirklichkeit in Einklang zu bringen ist: „Nach dem IPA liegt das ungespannte /a/ weiter vorn als das gespannte /a /. Ob es sich artikulatorisch im Deutschen tatsächlich so verhält, ist unsicher“ (EISENBERG 1998:32). Bei RAMERS / VATER (1991:116f.) und RAMERS (2001:32) reihen sich die $a$-Laute regulär ein in die Opposition gespannt/ungespannt.

Vgl. auch den Beitrag von KoHLER (1999:87) im IPA-Handbuch, in dem die deutschen a-Laute ebenso nur nach ihrer Quantität unterschieden werden: [a, a:].

13 Hinzu kommt der seltene, z.B. in der Interjektion pfui auftretende Diphthong, transkribiert als [pfui] (Duden) oder [pfur] (GWDA). - Einige Autoren vertreten 
hier behandelten Aussprachewörterbüchern finden wir diese Diphthonge in folgender Lautschrift vor:

mein neues Haus [main noyəs haus] Duden
mein neues Haus [magen nogøs hags] GWDA

Sowohl im Duden als auch im GWDA werden diese Diphthonge somit als aus zwei Vokalen bestehende Einzelphoneme dargestellt, was durch den verbindenden Bogen unter den konstitutiven Vokalen zum Ausdruck kommt. Als Begründung für den monophonematischen Charakter der Diphthonge wird angeführt, dass sie ,im Kontrast zu einem ihrer Bestandteile (in der Regel dem ersten) bedeutungsdifferenzierend auftreten, z.B. Bauch - Bach [baox - bax]“" (RAUSCH / RAUSCH 1993:104). Diese Auffassung ist jedoch nicht unumstritten, was der Duden in der Einführung anmerkt: „Es gibt auch Auffassungen, wonach die hier als Einzelphoneme betrachteten Diphthonge /ai au oy/ Phonemfolgen von je zwei Phonemen darstellen." (Duden 2005:35) Entsprechend dieser Auffassung wird letztlich die eigene, im Wörterbuchteil praktizierte Transkription in Frage gestellt, vgl. die folgende Fußnote im Duden (2005:36): „Phonetisch genauer wäre die Schreibung [aI au oY]]; andere schreiben dafür [ae ao əø] u.ä." Die zuletzt gezeigten Varianten (d.h. weder mit Bogen zur Kennzeichnung des monophonematischen Charakters noch mit untergesetztem Halbkreis zur Markierung des unsilbischen Charakters des zweiten Gliedes) ${ }^{14}$ sind jedoch kaum akzeptabel, da eine solche Transkription eine zweisilbige Realisierung nahelegt, und es ist ja ein Unterschied, ob von dem Landesnamen Laos ['la:os] oder von einer Laus [laus laos oder: launs laoss] die Rede ist. Die

zudem die Ansicht, dass auch die durch die $r$-Vokalisierung nach Vokal entstandenen Phänomene zu den Diphthongen zu rechnen sind. So nimmt z.B. VATER (2004:17), gestützt vor allem auf phonologische Untersuchungen, insgesamt „13 durch /r/-Glidisierung entstandene Diphthonge an, nämlich [iv, ye, ue, ev, øe, ov;

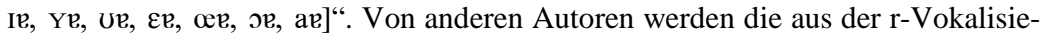
rung nach Vokal entstehenden einsilbigen Verbindungen entweder als „unechte“ Diphthonge bezeichnet (HAKKARAINEN 1995:62) oder ausdrücklich ,nicht zu den Diphthongen gezählt“ (zur Begründung vgl. u.a. MEINHOLD / STOCK 1982:86f.). Die in diesem Zusammenhang entstehenden Probleme für die IPA-Transkription werden im vorliegenden Aufsatz ebenfalls nicht im Abschnitt „Diphthonge“, sondern im Abschnitt „Vokalische und konsonantische Realisierung des $r^{\text {“ (s.u.) }}$ behandelt. Vgl. auch Anm. 16.

14 Besser macht es beispielsweise MiKoŁAJCZYK (2005:71), die sich zwar von den beteiligten Phonemen her auch an die GWDA-Kodifizierung hält [ae ao øø్n], jedoch das jeweils zweite Glied als unsilbisch markiert. 
monosyllabische Realisierung sollte auf jeden Fall in der Lautschrift erkennbar sein. $^{15}$

Das zweite Problem bei der Darstellung der deutschen Diphthonge in einem Aussprachewörterbuch ist die Qualität der beteiligten Vokale, namentlich des zweiten Gliedes. Sowohl im GWDA als auch im Duden suggeriert die Transkription, wir hätten es im zweiten Glied der Diphthonge jeweils mit gespannten Vokalen zu tun, noch dazu mit jeweils verschiedenen; vgl. im Duden [ai] [au] [oy] und im GWDA [ae] [ao] [oø]. Während das GWDA am gespannten Charakter des zweiten Vokals keine Zweifel aufkommen lässt, begibt sich der Duden auch hier in einen kreativen Widerspruch, vgl. die bereits oben zitierte Fußnote aus der Einleitung: „Phonetisch genauer wäre die Schreibung [aI au oYn].“ (Duden 2005:36) Diese Auffassung, wonach es sich bei dem jeweils zweiten Glied eben nicht um einen gespannten, sondern um einen ungespannten, unsilbischen Vokal handelt, kommt im Wörterverzeichnis nicht zum Ausdruck. Es spricht jedoch vieles dafür, dass es sich bei dem zweiten Glied in der Tat um einen unsilbischen, ungespannten Vokal handelt. Denn wie sollen die immer wieder von verschiedenen Autoren übereinstimmend konstatierten Merkmale der deutschen Diphthonge (u. a. Silbenakzent auf dem ersten Glied, Stärkeverminderung während der Gleitbewegung vom ersten zum zweiten Glied, Sinken der Lautstärke) realisiert werden, wenn während der Artikulation der Spannungsgrad erhöht wird, d.h. also von den ungespannten Phonemen [a] bzw. [0] hin zu den gespannten Phonemen [e], [o] und [ø] bzw. hin zu [i], [u] und [y]? Die Feststellung von RAUSCH / RAUSCH (1998:26), wonach in einer einsilbigen Zusammensetzung von Vokalen immer nur ein offener mit einem geschlossenen Vokal kombiniert werden kann, muss präzisiert werden, denn schon im GWDA, an dem sich die Autoren orientieren, finden sich - üb-

15 Verwiesen sei hier auch auf die von VATER (2005:33) gegebene Charakterisierung der Diphthonge. Danach handelt es sich bei einem Diphthong um eine ,,(der gleichen Silbe angehörende) Folge aus Vokal + Glide“. Ein Glide „hat (phonetisch) vokalische Eigenschaften, bildet aber (phonologisch) keinen Silbengipfel"“ (VATER 2005:33, Anm. 15). - Mitunter finden sich in der Literatur Widersprüche zwischen der korrekten Beschreibung und einer irreführenden Transkription der Diphthonge, vgl. etwa MoRcinIEC (1990), der die deutschen Diphthonge richtig als „einsilbige Vokalverbindungen“ beschreibt, deren zweites Glied ,als schwacher, unsilbiger Vokal" ausgesprochen wird (MoRCINIEC 1990:40, 14). Die von ihm verwendete Transkription [ae ao əø] ohne Sonderzeichen zur Markierung des unsilbischen Charakters des zweiten Gliedes wird dieser Beschreibung jedoch nicht gerecht., vgl. Beispiele wie Eile ['aelə], Raupe ['raopə], Eule ['øølə] (MORCINIEC 1990:41). 
rigens in Übereinstimmung mit dem Duden - Transkriptionen wie [re'gio:n] oder [glorino:la], in denen eine einsilbige Vokalkombination aus zwei geschlossenen, gespannten Vokalen auftritt. Bei der vokalischen Realisierung des $r$ nach langem Vokal in Wörtern wie Bier [bi:en], Uhr [u:e्s], Ohr [o:en] handelt es sich um einsilbige Vokalkombinationen aus einem geschlossenen, langen, gespannten Vokal und einem schwachtonigen Vokal, der weder als „offen“ noch als „geschlossen“ bezeichnet werden kann. In der Duden-Transkription der

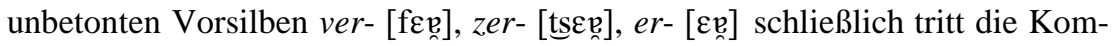
bination eines offenen, kurzen, ungespannten Vokals mit einem schwachtonigen Vokal auf, und die 2005 erschienene 6. Auflage des Duden-Aussprachewörterbuches bezeichnet bereits generell die vokalische, unsilbische Realisierung des $r$ nach kurzen, offenen Vokalen am Wortende oder vor Konsonant als Bestandteil der „genormten Lautung“, verwendet allerdings in der Lautschrift des Wörterverzeichnisses die konsonantische Realisierung, vgl. Wörter wie örtlich ['œrtlıç, 'œẹtlıç] und dürr [dyr, dYẹ] (Duden 2005:54). ${ }^{16}$

Die Möglichkeiten einsilbiger Vokalkombinationen sind somit sehr vielfältig, und es spricht nichts dagegen, bei der Kodifizierung der Diphthonge $e i$, $a u, e u$ - wie in der Fußnote der Duden-Einleitung - von einer Kombination zweier ungespannter Vokale auszugehen, wobei der zweite Vokal als unsilbisch zu markieren ist: [aI au or]]. Was die Höhe des zweiten Vokals anbelangt, so liegt dieser Vorschlag aus der Duden-Einleitung genau zwischen der im DudenWörterbuchteil praktizierten Transkription und der Transkription des GWDA

16 VATER (2004, 2005:33f.) beschreibt - wie oben erwähnt - die vokalisierten Varianten prinzipiell als in den Bereich der Diphthonge gehörend. Er betrachtet diese ,neuen“ Diphthonge allerdings im Unterschied zu den zugrundeliegenden Hauptdiphthongen [aI au oY̌] als aus Vokal $+r$ abgeleitete Diphthonge (VATER 2004:15f.). Für die Aussprache ist dies unerheblich, denn das vokalische $r$ verändert den Klang des vorausgehenden Vokals ,wie in einem Diphthong“ (HIRSCHFELD / REINKE / StOck 2007:165). Allerdings liefert dieser Statusunterschied (zugrundeliegend/abgeleitet) offenbar Argumente für die erwähnten, hier nicht zu entscheidenden Diskrepanzen in der Diphthongfrage. Man vergleiche das englische Wortpaar hear - hearing mit dt. hör - hören. Während der unstrittige Diphthong [Iə] in dem (britisch)-englischen Beispiel auch beim Übergang des $r$ zur Folgesilbe erhalten bleibt, also: [hı] / ['hı.rin], liegt der Fall im Deutschen anders: Aus [hø:! $]$ ] wird nicht etwa *['hø:్rən], sondern ['hø:rən]. Vgl. auch den (britisch)-englischen Diphthong [eə] in Wörtern wie fair [feə] und fairy ['feə.ri] (nach RoAch / HARTMAn / SETTER 2006) im Kontrast zu dem eingedeutschten fair [fe: sprochen wird. 
GWDA vs. Duden-Aussprachewörterbuch

und trifft auch unter diesem Aspekt die phonetische Realität am ehesten, vgl.

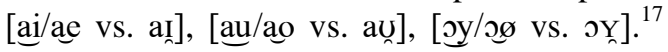

\section{Vokalquantität}

Im Gegensatz z. B. zum Polnischen ist die Klangqualität der Vokale durch die Opposition gespannt/ungespannt charakterisiert. Während ungespannte Vokale - zumindest in der Standardaussprache - stets kurz realisiert werden ${ }^{18}$, ist die Quantität der gespannten Vokalphoneme - offenbar in Abhängigkeit vom Akzent, entweder lang oder kurz, so z. B. in Fremdwörtern wie Musik [mu'zi:k] vs. Musiker ['mu:zike].$^{19}$ Es ergeben sich somit für das Deutsche folgende Vokalpaare: gespannte Vokale (lang/kurz) [i/ $/ \mathrm{i}, \mathrm{y:} / \mathrm{y}, \mathrm{u} / \mathrm{u}, \mathrm{e}: / \mathrm{e}, \varnothing: / \varnothing$, o: $/ \mathrm{o}]$ versus ungespannte Vokale (stets kurz) $[\mathrm{I}, \mathrm{Y}, U, \varepsilon, \propto$, o] $+[\varepsilon]$ (Ausnahme: ungespannt lang). Die Sonderstellung der $a$-Laute ist oben erörtert worden, hier betrifft die Opposition ausschließlich die Quantität: [a: / a]. Die schwachtoni-

17 Bei monophonematischer Interpretation der Diphthonge ist natürlich auch [aI au ơy] denkbar (so z.B. bei DoMIŃCZAK 1993:98, 297). Aus didaktischen Gründen problematisch könnten sich hingegen die von VATER (2005:33) vorgeschlagenen Transkriptionen [aj], [oj], [aw] erweisen. Damit würden - zumindest bei polnischen Lernern - Interferenzfehler möglicherweise unterstützt, vgl. die IPA-Transkription polnischer Lexeme wie frajer ['frajer], majster ['majster]; troje ['troje], kojce ['kojtse]; auto ['awto], aula ['awla] - deren korrekte Aussprache sich deutlich von den formal vergleichbaren deutschen Lexemen Freier, Meister; Treue, Käuze; Auto, Aula unterscheiden sollte. Siehe dazu die kontrastiven Hinweise bei MorCinIEC / PRĘDOTA (2005:129-132) und DoMiŃCZAK (1993:98-107).

18 Eine Ausnahme ist das [ $\varepsilon$ :], ein langer, ungespannter Vokal, der nicht nur in der Umgangslautung von großen Teilen der deutschen Sprachgemeinschaft durch [e:] ersetzt wird, also ['ne:mlıç] statt ['ne:mlıç]. TERNES (1999:94) sieht sich dadurch sogar veranlasst, zwei Aussprachenormen des Deutschen gelten zu lassen: „1. eine

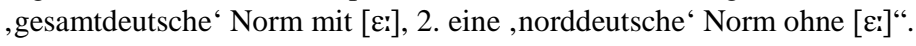

RAMERS (2001:32) weist darauf hin, dass die gezeigte Abhängigkeit der Länge von der Betonung ,nur für gespannte Vokale vor der Wortakzentstelle“ gilt, vgl. Musik. „Unbetonte gespannte Vokale nach dem Wortakzent können dagegen auch lang realisiert werden." (RAMERS 2001:33) Als Beispiele werden u.a. genannt Heimat ['haima:t], Almosen ['almo:zn], Schicksal ['Srkza:1]. Insgesamt sei das Verhältnis von Gespanntheit und Länge im deutschen Vokalsystem „,noch keineswegs ausreichend geklärt" (RAMERS 2001:33). Sicher scheint, dass bei der Klärung dieser Frage eine Differenzierung zwischen deutschstämmigen Wörtern einerseits und Fremdwörtern andererseits erfolgen muss, vgl. MEINHOLD / STOCK (1982:80f., 89f.). 
gen Vokale [ə] und [e] treten grundsätzlich nicht in langer Quantität auf. Zur wirklich messbaren Dauer der Vokale heißt es im GWDA

Die absolute Dauer langer und kurzer Vokale hängt in starkem Maße vom Grad der Beschwerung des Wortes innerhalb der Sprechsituation sowie vom Sprechtempo ab. Lange Vokale in unbetonter Stellung sind der absoluten Dauer nach im allgemeinen etwas kürzer als in betonter Stellung (z.B. ausgesöhnt neben versöhnen); die Erfordernisse einer Sprechsituation können Dehnungen hervorrufen. Eine genaue Differenzierung der Vokalqualität läßt sich deshalb bei der Aufzeichnung der Aussprache nicht durchführen. Im allgemeinen werden aus diesem Grunde nur lange und kurze Vokale unterschieden. (GWDA 1982:27)

Dennoch hat man sich im GWDA in zwei Fällen dazu entschieden, eine Kategorie „halblanger Vokale“ anzugeben: 1. bei volltonigem, unbetontem Vokal im Auslaut (Wörter wie Anna, Kino oder Emu); 2. „in der ersten Silbe eingedeutschter Wörter, wenn sie offen ist und mindestens vier Silben vor der Akzentsilbe steht“ (Wörter wie Meteorologie) (GWDA 1982:27). Zur Begründung wird angeführt, in Wörtern wie Anna usw. solle durch die Angabe der halben Länge eine ,,übermäßige Längung des auslautenden Vokals“ verhindert werden, in Wörtern wie Meteorologie gehe es darum, „durch geringe Dehnung des Vokals eine erforderliche Nebenakzentuierung zu kennzeichnen“ (GWDA 1982:27). Unverständlich bleibt, warum z. B. in dem Namen Anna laut GWDA ein halblanger Vokal zu sprechen ist ['ana'], in dem zusammengesetzten Ortsnamen Annaberg ['anaberk] (vgl. GWDA 1982:170) hingegen nicht, obwohl das erste Namenglied Anna hier natürlich auch einen volltonigen, unbetonten Vokal im Auslaut hat, der durch das zweite Glied Berg keine weitere Kürzung erfährt. Die Einrichtung einer dritten Vokalquantität, nämlich der der halblangen Vokale, erleichtert nicht das Verständnis des deutschen Vokalsystems, namentlich für Sprecher von Ausgangssprachen, deren Vokale hinsichtlich der Quantität keine bedeutungsunterscheidende Differenzierung zeigen. So ist es für polnische Sprecher, deren muttersprachliches Vokalsystem keine bedeutungsunterscheidenden Quantitätsunterschiede kennt, eher verwirrend, plötzlich mit halblangen Vokalen konfrontiert zu werden. In Namen wie Bruno, Gero, Otto usw. ist nicht die Quantität des auslautenden Vokals entscheidend (denn die unterliegt wegen des fehlenden Akzentes sowieso der Tendenz zur Kürzung), sondern die Qualität. Der zu realisierende $o$-Laut [o] muss geschlossen und gespannt sein, und so ein $o$-Laut unterscheidet sich letztlich auch in der Quantität nicht von einem $o$-Laut, wie er etwa in nichtakzentuierten Silben von Fremdwörtern realisiert wird, wo ihn auch das GWDA als kurz, gespannt und geschlossen angibt, vgl. Wörter wie Hotel [ho'tel], Prophet [pro'fe:t]. 
GWDA vs. Duden-Aussprachewörterbuch

Das einzige Phonetik-Lehrbuch, das die ,halblangen Vokale“ systematisch mit darstellt, ist RAUSCH / RAUSCH (1998). Auch MiKOŁAJCZYK (2005:60, 63) verwendet „halblange Vokale“, ohne dies allerdings zu erläutern. In den anderen hier interessierenden Lehr- und Übungsbüchern kommt die Kategorie der „halblangen Vokale“ nicht vor. Die Quantität des gespannten $o$-Lautes in Wörtern wie Auto, Kino und Professor wird übereinstimmend als kurz beschrieben (vgl. u. a. MORCINIEC / PRĘDOTA 2005:123). Auch im Duden-Aussprachewörterbuch (Duden 2005) treten kurze, gespannte Vokale sowohl in nichtakzentuierten Silben in Fremdwörtern als auch bei volltonigem, unbetontem Vokal im Auslaut auf. „Halblange Vokale” werden im Duden gar nicht erst erwähnt, was aus didaktischer Sicht sinnvoll erscheint.

\section{Vokalische/konsonantische Realisierung des $r$}

Für die Darstellung der vokalischen Realisierung des $r$ verwenden sowohl das GWDA als auch der Duden dasselbe IPA-Symbol: [e]. Dieser Vokal kann entweder silbisch (d.h. silbentragend) oder unsilbisch sein. Ist das vokalische $r$ Silbenträger, unterscheidet sich die Darstellung in den beiden Aussprachewörterbüchern nicht, vgl. Beispiele wie Lehrer ['le:re] oder klappern ['klapen]. Unterschiede gibt es in der graphischen Darstellung des unsilbischen $[\mathfrak{e}]$ im Silbenauslaut nach langem Vokal, vgl. u.a. das Wort Bier: GWDA [bi: $\left.{ }^{\mathrm{p}}\right]$ und Duden [bi:zer]. Zur Begründung des Unterschieds zwischen ['le:rr] einerseits und $\left[\mathrm{bi}^{\mathrm{e}}{ }^{\mathrm{p}}\right]$ andererseits heißt es im GWDA:

Der für $r$ substituierte Vokal wird auditiv als kürzer empfunden als der für die Folge er realisierte Vokal. Aus diesem Grunde wird die vokalische Substituierung des $r$ (nach langen Vokalen) durch Hochstellung des [ $\left.{ }^{p}\right]$ gegenüber dem vokalischen Monophthong von er [...] durch Normalschreibung des [e] kenntlich gemacht. Diese Differenzierung ist notwendig, um artikulatorisch gleichlautende, aber in der Bedeutung unterschiedliche Wörter untereinander als auch gegenüber Wörtern mit [ə] zu unterscheiden. (GWDA 1982:53f.)

Als Beispiel wird u.a. angeführt: hör' [hø:" $]$ - höher [hø:飞] - Höhe [hø:ə] (GWDA 1982:54). Die ,auditiv kürzere Wahrnehmung“ in hör' beruht indes auf dem unsilbischen Charakter des $[\mathfrak{e}]$ im Unterschied zum silbischen Charakter des $[\mathfrak{e}]$ in höher. ${ }^{20}$ Es liegt also nahe, diesen Kontrast durch das entspre-

20 Zur Diphthongdiskussion in diesem Zusammenhang vgl. die Anmerkungen 13 und 16. Das Problem der graphischen Darstellung des silbischen oder unsilbischen Charakters des $[\mathrm{e}]$ in der Transkription ist jedoch weniger ein theoretisches denn ein praktisches. Entscheidend ist es, mit Hilfe der IPA-Transkription die 
chende diakritische IPA-Zeichen zur Markierung des nichtsilbischen Charakters darzustellen, wie es der Duden tut. Diese Lösung ist auch graphisch die einfachere, vgl.: hör' [hø:e] - höher [hø:e] - Höhe [hø:ə] (Duden). Auch neuere Darstellungen verwenden hochgestelltes $\left[^{\mathrm{b}}\right]$ in der Regel nicht mehr (vgl. MiKoŁAJCZYK 2005:59, MORCINIEC / PRĘDOTA 2005:250, DIELING / HIRSCHFELD 2000:40, 196). ${ }^{21}$ Vokalisch realisiert wird auch die Buchstabenfolge <er> in den unbetonten Vorsilben <er-, ver-, zer-, her->, vgl. die Transkription der Wörter erlaufen, verlaufen, zerlaufen, herbei im GWDA und im Duden: [e'laofn], [fe'laofn], [tse'laofn], [he'bae] (GWDA); [عe'laufn], [feve'laufn] ], [tssev'laufn], [hev'bai] (Duden). Hier betreffen die Unterschiede in der Transkription lediglich den Grad der Abschwächung der unbetonten Silbe. Ob also [fer-] oder [fe-] gesprochen wird, hängt von der Stärke der Akzentuierung in der nächstfolgenden Silbe und vom Sprechtempo ab, vgl. dazu MEINHOLD (1973:33), der feststellt, dass eine volle Realisierung des /r/ auch auf der höchsten Formstufe als hyperkorrekt erscheint und somit für die Standardsprache nicht empfohlen werden kann. Eine Frage der Interpretation der lautlichen Wirklichkeit ist der Charakter des $r$ im Silbenauslaut nach langem $a$ Laut. Das GWDA - im Unterschied zu Wörtern wie Bier, leer, für, Öhr, Uhr, Ohr, führt, bohrt (in denen das $r$ vokalisch zu realisieren ist) - schreibt für das $r$ nach langem $a$ die konsonantische Realisierung vor, vgl. Wörter wie Haar [ha:r], klar [kla:r] - auch vor Konsonant: Fahrt [fa:rt], und schafft damit für die Folge „langes $a+r$ " eine Ausnahmeregel, die in der Sprechwirklichkeit nicht immer Bestätigung findet, denn eine volle konsonantische Realisierung ist vor allem bei langsamer und deutlicher Artikulation anzutreffen. RAUSCH / RAUSCH (1998:110), die sich in ihrer Darstellung ansonsten strikt an die GWDA-Kodifikation halten, äußern Zweifel an dieser Sonderregel und stellen fest, dass es der „Regeldurchgängigkeit“ sowie „einer unauffälligeren Aussprache“ zugute kommen könnte, wenn ,auch in dieser Position [d.h. nach langem $a$ - G.L.] vokalisiertes $r$ gesprochen oder eine Ersatzdehnung des langen $a$ realisiert werden würde““. ${ }^{22}$ Der sehr verbreiteten vokalischen Realisie-

Sprechwirklichkeit möglichst präzise, zugleich aber auch in didaktischer Hinsicht verständlich aufzuzeichnen.

21 Vgl. aber HiRSCHFELD / REINKE / STOCK (2007:119, 165), die daran festhalten, das vokalische $r$ in Wörtern wie Tür, Ohr usw. halbhoch zu setzen - [ty: ${ }^{\mathrm{p}}$, to: ${ }^{\mathrm{p}}$ ], im Unterschied zu Fällen, in denen das vokalische $r$ als Kern einer Silbe zu hören ist, wie z.B. in Vertreter [fe'tre:te]. vokalische $r$ häufig sogar vollständig getilgt wird, z.B. RAMERS / VATER 
rung von $r$ auch nach langem [a: $]^{23}$ trägt der Duden Rechnung, indem er innerhalb der genormten Lautung nach langem [a:] am Wortende oder vor Konsonant sowohl die vokalische als auch die konsonantische Realisierung zulässt, in der Lautschrift jedoch nur [en] verwendet (vgl. Duden 2005:54), z. B. Haar [ha:e] / [ha:r] oder Bart [ba:ett] / [ba:rt], im Wörterbuchteil aber ausschließlich [ha:e् ] und [ba:ett], analog zu Bier [bi:en], leer [le:e्र], für [fy:en], Öhr [ø:e्र], Uhr [u:e], Ohr [o:e], führt [fy:ent] und bohrt [bo:ent]. Am Beispiel der vokalischen Realisierung des $r$ lassen sich gut Entwicklungstendenzen ablesen, die sich in der deutschen Aussprache vollziehen und auf die letztlich auch ein Aussprachewörterbuch reagieren muss. Dies sei am Beispiel der $r$-Realisierung nach kurzem Vokal am Wortende oder vor Konsonant gezeigt, also in Wörtern wie Berlin, Form, Herr, örtlich. Während der verbreitete Ersatz von [r] durch unsilbisches [e], also [ํㅡ] , in diesen Fällen in der 3. Auflage des Duden-Aussprachewörterbuches (Duden 1990) noch im Kapitel „Ungenormte Lautung“ als Umgangslautung gekennzeichnet wurde (Duden 1990:58), ist dasselbe Phänomen seit der 4. Auflage im Abschnitt „Genormte Lautung” zu finden, vgl. Duden (2000:54) und Duden (2005:54), wo es heißt, dass nach den kurzen Vokalen [I $\varepsilon$ Y $\propto$ a $U$ o] am Wortende oder vor Konsonant konsonantisches $r$ [r], aber auch vokalisches $r$ [e $]$ vorkommt. In der Duden-Transkription wird jedoch in diesen Fällen ,im Allgemeinen“ nur [r] verwendet, z.B. Berlin [ber'li:n], Form [form], Herr [her], örtlich ['œrtlıç] - anstatt der vokalisierten, aber von der Duden-Redaktion immerhin schon als ,genormte Lautung“ aner-

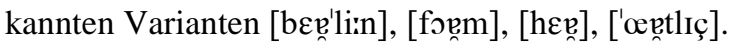

Zur Diskussion gestellt sei hier auch der Ansatz von HøYEM / ZICKFELDT (1992:98), die in Bezug auf Teilbereiche der beiden hier angesprochenen Problemfelder von einem ,intendierten [в]“" sprechen. Beschrieben wird dieses ,intendierte $[\mathrm{B}]^{\text {“ }}$ als ein Laut, der

(1991:111); Ergebnis ist eine Aussprache wie [ba:] / [ba:] für das Wort Bar. Die Ersatzdehnung besonders nach langem [a:] erwähnt auch HAKKARAINEN (1995: 63). In der Transkription kann diese Erscheinung durch doppelte Längenbezeichnung markiert werden: [ba::] (statt [ba:e $]$ ).

23 MeinHOLD (1973:33) merkt an, dass es nach [a: häufig zu Totalassimilationen ohne Kürzung des Vokals kommt. Gerade nach [a:] komme es jedoch, besonders bei Berufssprechern, auch zu „kompensatorische[n] Realisationen voller Allophone“. Generell gilt jedoch: „Nach Langvokal im Silbenauslaut ist die Vokalisation im Deutschen als obligatorisch anzusehen.“ (MeINHOLD 1973:32) 


\section{Gero Lietz}

[...] artikulatorisch zwischen dem stimmhaften, uvularen/velaren Spiranten [в] und der vokalischen Variante des /r/, [e], liegt. Dies ist ein R-Laut, der noch immer ein konsonantisches Element hat, bei dem eine spirantische Artikulation aber nur eingeleitet oder intendiert wird, so daß das Resultat ein sehr schwacher, fast schon vokalischer, Laut wird. Dieses [ $\mathrm{G}]$, das wir hier intendiertes [G] nennen wollen, ist stellungsgebunden, also ein kombinatorisches Allophon, da es nur nach kurzem Vokal vor stimmhaftem oder stimmlosem Konsonanten $\mathrm{V}+\mathrm{r}+\mathrm{K}$, z. B. $<$ Turm> [tus $s$ ] $],<$ Ort $>$ [orst $]$, oder nach langem [a: vor Konsonant, [a: $+\mathrm{r}+\mathrm{K}$, z. B. <Art> [arst] , <(ihr) wart> [va:st], gesprochen wird.

In den von HøYEM / ZICKFELDT beschriebenen Fällen lassen die neuesten Auflagen des Duden-Aussprachewörterbuches (vgl. oben) im Rahmen der genormten Lautung grundsätzlich sowohl die konsonantische als auch die vokalische Realisierung zu, mit der erwähnten unterschiedlichen Darstellungsweise im Wörterbuchteil, vgl. [a: rt] vs. [ort]. Zugleich erweitert der Duden die Entscheidungsfreiheit vokalische/konsonantische Realisierung auch auf die Fälle, in denen das $r$ im absoluten Silbenauslaut steht, vgl. die obigen Beispiele Haar und Herr, im Wörterbuchteil des Duden allerdings ebenfalls als [ha:e ] und [her] verzeichnet. Zu fragen ist, wie das Konzept des ,intendierten [в]“ nutzbar gemacht werden kann für die Kodifizierung der deutschen Aussprachenorm. Es fällt auf, dass der konsonantische Charakter des $r$ in Wörtern wie Haar oder (ihr) wart bei normalem Sprechtempo deutlich schwächer ausgeprägt ist als initial oder intervokalisch realisiertes konsonantisches $r$ (wie etwa in Rose oder hören). Ist also die ausschließlich konsonantische Realisierung [ha:r / va:rt], wie sie das GWDA festschreibt, gerechtfertigt? Andererseits darf die im Duden beschriebene standardsprachliche Tendenz einer wahlfreien konsonantischen oder vokalischen Realisierung von $r$ nach kurzem Vokal (dort, Form, Herr usw.) sicherlich nicht dazu führen, dass bei einer Weiterentwicklung der Kodifizierung der deutschen Standardaussprache die vokalische Realisierung den Vorrang erhält, also [dop̧t fợm hę्र] statt [dort form her]. Dies entspräche zwar der artikulatorischen Realität in weiten Teilen Norddeutschlands ${ }^{24}$, für die überregionale Standardlautung darf jedoch nicht vernachlässigt werden, dass es viele Sprecher gibt, die in den genannten Fällen sehr wohl einen deutlichen Konsonanten sprechen, sei es als Zungenspitzen- $r$ oder als uvularen/velaren Spiranten. Bei Gebrauch eines

24 Im Norddeutschen ist sogar ein vollständiger Verzicht auf die Realisierung des $r$ zu beobachten - zugunsten einer in der Standardsprache unzulässigen Längung des ungespannten Vokals, vgl. dort [dort] oder Form [form], analog übrigens zum britischen Englisch, vgl. engl. port [po:t] oder form [fo:m]. 
stimmlosen, uvularen/velaren Spiranten kann das sogar dazu führen, dass Wörter wie dort/Docht [doşt / doxt] oder warten/wachten ['vaştṇ / 'vaxtñ] auditiv kaum voneinander zu unterscheiden sind (vgl. HøYEM / ZICKFELDT 1992:95).

\section{Reduzierung der Endsilbe -en nach Verschlusslauten}

Die Realisierung der Endsilbe -en in der deutschen Standardaussprache geschieht in Abhängigkeit vom vorausgehenden Stammauslaut. Voll realisiert übereinstimmend nach dem GWDA und dem Duden - wird die Endsilbe -en nur nach vorausgehendem Vokal bzw. Diphthong, nach Nasalen, nach $l, r, j .{ }^{25}$ Nach Engelauten kommt es zur Schwa-Elision, gesprochen wird ein silbisches $n$, vgl. Beispiele wie hoffen ['hofn], Rosen ['ro:zn], waschen ['vafn], rächen $\left[\right.$ 'rrçn]. ${ }^{26}$ Auch in dieser Frage gibt es Einhelligkeit zwischen GWDA und Duden. Unterschiede in der Transkription sind nach Verschlusslauten feststellbar. Im GWDA heißt es, dass schwachtoniges $e$ in der Regel nach Verschlusslauten nicht gesprochen wird. Der Kontext kann indes eine volle Realisation erfordern: „Wenn es die Sprechsituation (Sprechtempo, Beschwerung des Wortes usw.) erfordert, wird es realisiert. Im Wörterverzeichnis stehen die vokallosen Formen an erster Stelle, die vollen (selteneren) Formen an zweiter Stelle." (GWDA 1982:35)

Es ergeben sich somit laut GWDA für die Realisierung von -en nach Verschlusslauten kontextabhängige Varianten: ['lo:bm] / ['lo:bən]; ['tro:pm] / ['tro:pən], ['ly:gy] / ['ly:gən]; ['dryky] / ['drykən]; ['fa:dṇ] / ['fa:dən]; ['tre:tn] / ['tre:tən]. Je nach Artikulationsstelle des vorausgehenden Verschlusslautes erfolgt bei reduzierter Endsilbe eine Assimilation, deren Ergebnis ein nasaler, silbischer Konsonant ist. Die Silbenzahl bleibt trotz Schwa-Elision unverändert.

In der Kodifizierung des Duden-Aussprachewörterbuches ist vor allem eine folgenreiche Inkonsequenz zu kritisieren. Im Kapitel „Genormte Lautung“

25 Beispiele sind Wörter wie bauen ['baüən], gehen ['ge:ən], kommen ['kəmən], kennen ['kenən], singen ['zıjən], knallen ['knalən], bohren ['bo:rən], Bojen ['bo:jən]. Vgl. die Übersicht zum Vorhandensein oder Ausfall des Schwa-Lautes bei sachlich-neutraler Rede (RAUSCH / RAUSCH 1998:224). Je nach Kontext (im Sinne von MEINHOLD 1973:70) kann es umgangssprachlich zu weitergehenden Reduktionen kommen, die bis zum Silbenverlust gehen, vgl. gehen [ge:n], kommen [kom:], kennen [ken:], singen [ziy:], knallen [knaln] oder bohren [bo:ennn].

26 Eine Ausnahme bildet das Diminutivsuffix -chen, bei dem volle Realisierung vorliegt, vgl. Mädchen ['me:tçən]. 
heißt es: „Statt [pn], [bn]], [kn], [gṇ] wird im Allgemeinen häufiger [pm], [bm], [ky], [gy] gesprochen.“ (Duden 2005:38) Im Wörterverzeichnis erscheinen trotz dieser zutreffenden Feststellung ausschließlich die in der Tabelle am Ende dieses Beitrages angegebenen Varianten ['lo:bn]], ['tro:pn], ['ly:gn], ['drykn], ['fa:dn], ['tre:tn]. Während nach $d$ und $t$ ein silbisches [n] phonetisch möglich ist (sowohl [d], [t] als auch [n] werden an den Alveolen gebildet, Assimilation der Artikulationsstelle ist die Folge) und empfehlenswert erscheint (das GWDA lässt hier im Unterschied zum Duden lediglich auch die unreduzierte Realisierung zu), ist die im Wörterverzeichnis des Duden angegebene Kodifikation [n] nach $b, p, g$ und $k$ artikulatorisch überhaupt nicht realisierbar, denn das alveolare [n] ist viel zu weit von den vorhergehenden Artikulationsstellen ( $b, p$ : bilabial; $g, k$ : velar) entfernt, als dass hier eine entsprechende Assimilation möglich wäre. Um von den Lippen bzw. dem Velum zum Zielpunkt des [n], den Alveolen, zu gelangen, ist eine Lösung der vorausgehenden Verschlusslaute nötig, wodurch es automatisch zur vollen Realisation (d.h. mit Schwa) kommt. Eine vokallose Realisation nach $b, p, g$ und $k$ ist indes nur nach der für diese Fälle im GWDA kodifizierten Art und Weise möglich, d.h. als [m] (nach $b, p$ ) bzw. [y] (nach $g, k$ ). Der Verschluss von [b], [p] bzw. [g] , [k] wird dann nicht oral, sondern nasal - unter Beibehaltung der ursprünglichen Verschlussstelle - gelöst. Die Verfahrensweise des Duden in dieser Frage ist offenbar ein Zugeständnis an die Schriftsprache. Bei allem Verständnis dafür, dass die phonetische Transkription natürlich für den Benutzer nicht unnötig kompliziert und damit auch noch lesbar bleiben muss, muss vor zu großer Vereinfachung gewarnt werden, insbesondere, wenn durch die Transkription artikulatorisch nicht realisierbare Lösungen postuliert werden.

\section{Weitere Unterschiede im Bereich [ə] in Endsilben}

Im GWDA gibt es vereinzelt Ausnahmereglungen für die Realisierung des Schwa-Lautes in Endsilben. Die Endung -el wird, so heißt es im GWDA (1982:36), u. a. nach [g] voll realisiert, vgl. Beispiele wie Prügel ['pry:gəl], Kegel ['ke:gəl], entsprechend prügeln ['pry:gəln], kegeln ['ke:gəln] u. a. Zugleich schreibt das GWDA jedoch Schwa-Elision mit lateraler Sprengung nach [k] vor, vgl. Fackel ['fakl], fackeln ['faklı]], Deckel ['dekl]]. Eine Begründung für diese inkonsequente Sonderregel wird nicht gegeben. Bereits in RAUSCH / RAUSCH $(1998: 224,227)$ werden die Lautverhältnisse nach $[\mathrm{g}]$ und $[\mathrm{k}]$ einheitlich mit Schwa-Elision und silbischem $l[1]$ dargestellt. Die Endsilbe -el wird demnach nur vor und nach Vokal voll realisiert, vgl. Prügel vs. Prügelei: ['pry:gl] vs. [pry:ga'lae] bzw. [pry:ga'lai]. Auch der Duden (2005:40) kommt 
ohne Sonderregel für -el nach [g] aus. Hinzu tritt - und hier besteht Übereinstimmung zwischen Duden und GWDA, die volle Realisierung der Endsilbe -el nach [r], vgl. Barrel ['berəl]. In allen anderen Fällen (nach Verschlusslauten, nach Engelauten, nach Nasalen) fällt [ə] aus, und ein silbisches $l[1]$ schließt sich an.

Eine weitere Ausnahme macht das GWDA - im Unterschied zum Duden - für die Endsilbenverbindung -igen. Hier soll laut GWDA die Endsilbe -en stets voll, d.h. als [ən] realisiert werden. Die sonst im GWDA nach dem Stammauslaut [g] übliche Variante mit Schwa-Elision und dem silbischen Nasal [y] ist in diesem Fall nicht vorgesehen. Das Duden-Aussprachewörterbuch trifft auch hier keine besondere Regelung, vgl. das Wort berichtigen: [bə'riçtrgən] (GWDA) vs. [ba'riçtıgn $]^{27}$ (Duden).

\section{Affrikaten}

Die umstrittene phonologische Klassifizierung der Affrikaten im Deutschen ${ }^{28}$, d.h. die Frage nach ihrer monophonematischen oder ihrer biphonematischen Wertung, soll hier nicht Gegenstand der Diskussion sein. ${ }^{29}$ Auch wenn die Affrikaten-Frage bzw. deren eventuelle Lösung „für die Aussprache des Deutschen keine Konsequenzen" (HAKKARAINEN 1995:85) hat, so hat doch die Kontroverse ihren Niederschlag in der IPA-Transkription der hier untersuchten Aussprachewörterbücher gefunden. Während das GWDA von biphonematischen Explosiv-Frikativ-Lautverbindungen ausgeht (vgl. Pfad [pfa:t], Zug [tsu:k], Tscheche ['t $\mathrm{f} \varepsilon c ̧ ə]$, Gin [dzIn]), geht der Duden von der monophonematischen Interpretation aus, in der dieselben Beispielwörter wie folgt transkri-

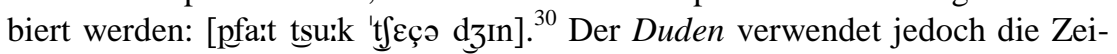

27 Richtig wäre auch hier natürlich die assimilierte Variante [bo'rrçtıgy] mit silbischem [y] statt [n], vgl. die Ausführungen oben. Vgl. stellvertretend BUSSMANN (2002:53f.), KOHLER (1995:166), RAMERS / VATER (1991:85-91), MEINHOLD/ STOCK 1982:127-129).

29 Auch in anderen Sprachen sorgt der Status von Affrikaten für Diskussionsstoff, vgl. für das Englische CRutTEnden (1994:157-162) und RoACH / HARTMAN / SETTER (2006:11).

Verschiedene Autoren verweisen darauf, dass auch noch weitere Konsonantenverbindungen als Affrikaten interpretierbar sind, u.a. die Kombination stimmloses [6] nach [k] (wie z.B. in Kreis oder Kraft) sowie die in der schweizerdeutschen Mundart anzutreffende Realisierung [kx], wie z.B. in Kind [kxint] (vgl. HøYEM / ZICKFELDT 1992:91). Zudem wird darauf hingewiesen, dass auch Explosiv-Frika- 


\section{Gero Lietz}

chenkombinationen [pf ts t $\mathrm{t} d 3$ ] „nicht für fremdsprachliche Aussprache“ (Duden 2005:12). Das führt dazu, dass Affrikaten bei der Angabe einer eingedeutschten Variante durch Bogen gekennzeichnet werden; bei der Angabe der fremdsprachlichen Lautung entfällt der Bogen hingegen, vgl. Chesterfield (Mantel) ['t $f$ Estefi:lt], aber Chesterfield (Name) ['t $\mathrm{t} \varepsilon s t ə f i: l d] ;$ Jersey (Stoff) ['dzø:ęzi], aber Jersey (Name) ['dzə:zI]. Das GWDA verzichtet generell auf den verbindenden Bogen und damit auch auf eine Differenzierung dieser Art. Eine Frage für den Phonetik- und Transkriptionsunterricht ist, ob in der Transkription der deutschen Standardaussprache generell auf die Affrikatenbögen verzichtet werden könnte. Ein Argument für den Verzicht wäre die Tatsache, dass von der phonetischen Realisierung her kaum Differenzen zwischen den oben erwähnten eindeutschenden und den fremdsprachlichen Lautungen feststellbar sind, das heißt also: Akustisch gibt es zwischen dem [t $\mathrm{f}]$ in Chesterfield als Mantel keinen Unterschied zu dem [ $\mathrm{t}]$ ] in dem englisch artikulierten Namen Chesterfield. ${ }^{31}$ In beiden Fällen werden die Konsonantenverbindungen als artikulatorische Einheit realisiert. - Einbezogen werden in die Betrachtungen muss auch das Aufeinandertreffen der an den Affrikaten beteiligten Explosivund Frikativlaute an der Morphemgrenze, z. B. in hat schon ['hat So:n], hat Sex [hat 'scks] oder abfüllen ['apfylən]. Bei langsamem Sprechen kommt der akustische Unterschied zwischen hat schon vs. Matsch, hat Sex vs. Hatz und abfüllen vs. Apfel vor allem in einem vergleichsweise längeren Friktionsgeräusch der Engelaute in hat schon, hat Sex und abfüllen zum Ausdruck. ${ }^{32}$ Bei

tiv-Verbindungen existieren, die im Unterschied zu den klassischen Affrikaten nicht an der gleichen Artikulationsstelle realisiert werden, aber dennoch ,,als Einheit artikuliert und deshalb zum Teil mit nur einem Buchstaben wiedergegeben [werden]“" (STOCK / HIRSCHFELD 1996:167). Genannt werden die Verbindungen [ks] und [kv], wie in Text und Quadrat.

31 Im Englischen wird bei der Transkription der Affrikaten generell auf den Gebrauch von Bögen verzichtet, obwohl der Affrikaten-Status zumindest von [t $\int$ ] und [dz] allgemein anerkannt wird: ,It is usual to regard $/ \mathrm{t} f /$ and $/ \mathrm{d}_{3} /$ as affricate phonemes in English; /ts dz tr dr/ also occur in English but are not usually regarded as affricate phonemes, but are treated as clusters." (ROACH / HARTMAN / SETTER 2006:11)

Vgl. auch entsprechende Beispiele für das Englische: „The two phrases why choose and white shoes are said to show the difference between the /t $\mathrm{J} /$ affricate (in the first example) and separate /t/ ans / $/$ / (in the second)." (ROACH / HARTMAN / SETTER 2006:11). CRUTTENDEN (1994:157) unterscheidet „,close-knit stop“ in dem Wort butcher von "disjunct stop“ in dem Wort lightship. „[T]he fricative is 
schnellerem Sprechtempo unterscheidet sich jedoch die kompakte Realisierung der Lautverbindungen an der Morphemgrenze akustisch kaum mehr von den Affrikaten in Matsch [t $/ \mathrm{t}$ ], $\mathrm{Hatz}$ [ts / ts] oder Apfel [pf / pf]. Wollte man jedoch solche Fälle mit Affrikatenbogen transkribieren, brächte dies erhebliche Probleme für die Markierung der Silbengrenze mit sich, denn die Silbengrenze kann in den mit Bogen dargestellten Affrikaten schwerlich innerhalb ein und desselben Phonems verlaufen ['ap-fy-lən], nicht richtig wäre zudem *['a-pfy-lən]. Korrekt darstellbar ist die Silbengrenze nur bei Verzicht auf den Bogen, also z.B. ['ap-fY-lən]. Eine andere Frage ist die nach der orthographischen Silbentrennung. Im Deutschen werden die für eine Affrikate stehenden Buchstaben grundsätzlich getrennt: Ap-fel, klat-schen, het-zen, Pid-gin.

Erweitert man den Blick auf andere Sprachen, wird deutlich, dass die Frage der Affrikaten (sowohl phonologisch als auch transkriptionspraktisch) immer einzelsprachspezifisch gelöst werden muss. So sind beispielsweise im Polnischen die Unterschiede zwischen den aus zwei Phonemen bestehenden Explosiv-Frikativ-Verbindungen und monophonematischen Affrikaten sowohl phonologisch als auch phonetisch relevant, worauf u. a. MORCINIEC (1990:92f.) in seiner kontrastiven Studie zum Lautsystem des Deutschen und Polnischen hinweist. Wesentliche Unterschiede in der phonetischen Realisierung (und auch in der Bedeutung!) weisen u. a. Minimalpaare wie Czech vs. trzech, czy vs. trzy oder dżemy vs. drzemy auf. Das Setzen von Bögen in der IPATranskription zur Unterscheidung zwischen Lautverbindungen und echten Affrikaten ist somit für eine Sprache wie das Polnische absolut notwendig. Eine

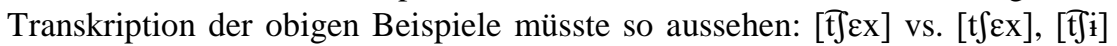

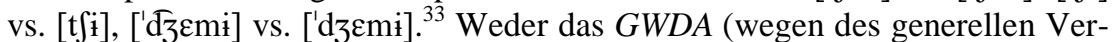
zichts auf Affrikatenbögen) noch der Duden (der die Affrikatenbögen auf deutsche Wörter und eingedeutschte Lautungen beschränkt) sind in der Lage, diese Unterschiede entsprechend zu verdeutlichen, vgl. im GWDA die polni-

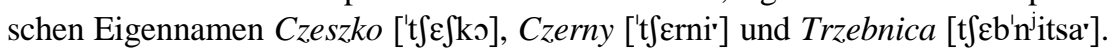

shorter in close-knit sequences: thus the friction in butcher is of shorter duration than the friction in lightship." (CRUTTENDEN 1994:158)

33 Für das Polnische ist traditionell die slawistische Transkription sehr verbreitet, die sich von der IPA-Transkription deutlich unterscheidet. In sprachwissenschaftlichen Publikationen begegnet man jedoch auch für das Polnische immer häufiger der IPA-Transkription. Eine Übersicht über beide Transkriptionsalphabete - das slawistische und das internationale - findet sich bei MADELSKA / WITASZEKSAMBORSKA (1998:13-20), einer Übungssammlung für Polonistikstudierende. Das Buch enthält auch sehr instruktive polnische Beispieltexte in beiden Transkriptionskonventionen. 
Der Duden versucht die Lautfolge $\operatorname{trz}$ wie folgt von der Affrikate $c z$ abzu-

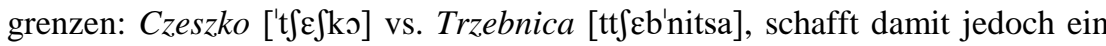
neues Problem, denn die Transkription [ttf] für trz steht mit der Sprechrealität des Polnischen nicht im Einklang. ${ }^{34}$

\section{Stimmritzenverschlusslaut}

Der Stimmritzenverschlusslaut [l], auch Glottalstopp, Glottisschlag oder Knacklaut genannt, kennzeichnet den Vokalneueinsatz (den ,festen Vokaleinsatz“ im Gegensatz zum ,gehauchten Vokaleinsatz“ [h], vgl. dazu RAUSCH / RAUSCH 1998:325-331). Er ist „,kein eigentliches Konsonantenphonem, sondern ein Grenzsignal. Er signalisiert vor Vokal den Wortanfang und die Fuge in Präfixbildungen und zusammengesetzten Wörtern, z. B. anekeln ['|an|e:kln], beachten [ba'|axtn]]“ Duden (2005:43). ${ }^{35}$ In Bezug auf den Stimmritzenverschlusslaut lassen sich in den hier betrachteten Aussprachewörterbüchern insbesondere zwei Unterschiede feststellen. Der erste betrifft die Markierung der Position des Knacklautes im Verhältnis zum Akzentzeichen. Während der Glottisverschlusslaut [l] im Duden-Aussprachewörterbuch hinter dem Akzentzeichen platziert wird, steht er im GWDA vor dem Akzentzeichen, vgl. z. B. die Wörter verächtlich und erinnern: Im Duden lautet die Transkription

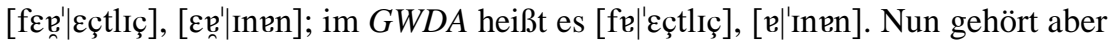
der den Vokal einleitende Knacklaut ebenfalls bereits zu der akzentuierten Silbe, er signalisiert ihren Anfang. Auch wenn der Knacklaut, wie erwähnt, „kein eigentliches Konsonantenphonem“ ist, trägt er die Merkmale eines Verschlusslautes. Wenn also ein Lexem wie bekannt (die zweite Silbe beginnt mit dem Verschlusslaut $[\mathrm{k}]$ ) die Transkription [bə'kant] hat ${ }^{36}$, besteht kein Anlass, im Falle des Glottisverschlusslautes anders zu verfahren. Zudem wird auch der gehauchte Vokaleinsatz [h] in beiden Wörterbüchern übereinstimmend als

34 Da in der Aussprache der polnischen Lautverbindung trz nicht das $t$ länger (oder doppelt) ausgesprochen wird, sondern in der Regel die Friktion des Engelautes länger erscheint als in der Affrikate $c z$, trifft die von MORCINIEC (1990:93) vorgeschlagene Transkription [t $\left.\iint\right]$ die Sprechrealität besser. Am plausibelsten ist jedoch der Gebrauch von Affrikatenbögen für $c z$, in Abgrenzung zur Lautverbindung trz, die ohne Bogen transkribiert wird.

35 Vor Vokal am Wortanfang wird in der Transkription das IPA-Symbol für den Stimmritzenverschlusslaut in aller Regel weggelassen.

36 Falsch wäre die Platzierung des Verschlusslautes [k] vor dem Akzentzeichen: *[bək'ant]. 
hinter dem Akzentzeichen stehend gekennzeichnet, vgl. verhalten [feęhaltn]] / [fe'haltṇ], Gehöft [gə'hœft]. Was liegt näher, als die ähnlichen, nur mit festem Vokaleinsatz zu sprechenden Wörter veralten und geöffnet analog zu behandeln? Plausibler als die im GWDA vorgenommene Trennung zwischen Glottisverschlusslaut und akzentuierter Silbe (vgl. [fe|'altn]], [gə|'œfnət]) erscheint die im Duden gewählte Verfahrensweise, in der eindeutig die Zugehörigkeit des Knacklautes zur akzentuierten Silbe zum Ausdruck kommt: [feę |altn]], [go'|œfnot].

Ein zweites Problem ist der Umfang der mit Glottisverschlusslaut zu sprechenden Wörter. Abweichende Festlegungen sind insbesondere im Zusammenhang mit akzentuierten Silben im Wortinnern von Fremdwörtern zu beobachten. Während der Duden in Wörtern wie Theater, museal, negroid, Atheismus eine kontinuierliche Weiterführung des Stimmtons favorisiert und somit auf den Glottisschlag verzichtet (vgl. die Transkriptionen [tea:te, muze'a:l, negro'iit, ate'ssmus]), schreibt das GWDA in den gleichen Wörtern den Glottisschlag vor: [te|'a:te, muze|'a:l, negro|'it, ate|'Ismus]. Welcher dieser Varianten der Vorzug gegeben wird, hängt maßgeblich vom Kontext einer Äußerung ab (Sprechsituation, Sprechtempo, Deutlichkeit der Artikulation). Auch sind regionale Unterschiede bei der Realisierung des Stimmritzenverschlusslautes $\mathrm{zu}$ beachten (besonders zwischen dem Nord- und dem Süddeutschen, vgl. HøYEM / ZICKFELDT 1992:73f.).

\section{Stimmtonverlust an der Wort- und Silbengrenze}

Beim Aufeinandertreffen von Konsonanten kommt es unter bestimmten Bedingungen zu Assimilationserscheinungen, die verschiedenen Gesetzmäßigkeiten folgen. So unterscheiden sich z.B. im Polnischen und im Deutschen die Gesetzmäßigkeiten für die Veränderung der Stimmhaftigkeit von Konsonanten an der Wort- und Silbengrenze. Phonetische Fehler in diesem Bereich halten sich selbst bei fortgeschrittenen Sprechern sehr hartnäckig. In aller Kürze fasst DIELING (1992) das Problem zusammen:

Während im Deutschen durchgängig progressiv assimiliert wird, sind die Assimilationsvorgänge im Polnischen überwiegend regressiv. So werden z. B. stimmlose Konsonanten vor stimmhaften meist stimmhaft. Damit sind Wörter vom Typ Erdgas, Nachbar, Dresden, wo diese Konstellation eintritt, von vornherein exponiert und deshalb zu beachten. (DIELING 1992:99)

Im Deutschen bleiben die stimmlosen Konsonanten (auch die auslautverhärteten) grundsätzlich stimmlos, unabhängig vom nachfolgenden Konsonanten. Im Polnischen hingegen werden stimmlose Konsonanten im Silbenauslaut immer 


\section{Gero Lietz}

dann stimmhaft, wenn an zweiter Position ein stimmhafter Konsonant folgt. Während also die Auslautverhärtung im Deutschen stets gültig ist, wird sie im Polnischen (wo sie grundsätzlich auch existiert) unter bestimmten Bedingungen außer Kraft gesetzt. Man vergleiche die folgenden Beispiele, in denen vergleichbare Konsonantenkonstellationen für das Sprachenpaar Deutsch/Polnisch aufgelistet $\operatorname{sind}^{37}$ :

\begin{tabular}{|c|c|c|c|c|}
\hline Polnisch & Transkription & Deutsch & Transkription & Interferenz \\
\hline $\begin{array}{l}\text { mów do mnie } \\
\text { Józef da }\end{array}$ & $\begin{array}{l}{\left[\begin{array}{ll}\text { muv do] } \\
{[j u z \varepsilon v} & d a\end{array}\right]}\end{array}$ & $\begin{array}{l}\text { Gustav der V. } \\
\text { kauf dir was }\end{array}$ & $\begin{array}{l}\text { [gustaf de:er] } \\
\text { [kauf di:en] }\end{array}$ & $\begin{array}{l}\text { [gustav de:e] } \\
\text { [kauv di:en] }\end{array}$ \\
\hline mów tato & [muf tato] & Gustav tut & [gustaf tu:t] & ----------------- \\
\hline $\begin{array}{l}\text { Bob zezuje } \\
\text { łap Zenka }\end{array}$ & $\begin{array}{l}{\left[\begin{array}{ll}\text { bob } & \text { zezuje] }\end{array}\right.} \\
{[\text { wab }} \\
\text { zenka] }\end{array}$ & $\begin{array}{l}\text { Absicht } \\
\text { schlapp sein }\end{array}$ & $\begin{array}{l}\text { ['apzictct] } \\
\text { [Slap zain] }\end{array}$ & $\begin{array}{l}\text { [abziçt] } \\
\text { [Slab zain] }\end{array}$ \\
\hline łap Tomka & [wap tomka] & Abteil & [ap'tail] & -------------- \\
\hline kod banku & [kod banku] & Erdbeben & [e:prtbo:bm] & [e:prdbe:bm] \\
\hline kod pocztowy & [kot pođTtəvi] & Nordpol & ['nortpo:l] & ------------------- \\
\hline
\end{tabular}

Interferenzgefahr für polnische Sprecher besteht immer dann, wenn an der Wort- oder Silbengrenze die zweite Position einer Konsonantenkontaktstelle mit einem stimmhaften Konsonanten besetzt ist. Polnische Sprecher sind dann - entsprechend ihren muttersprachlichen Gewohnheiten - versucht, auch im Deutschen die gesamte Verbindung stimmhaft zu sprechen. Im Deutschen hingegen ist die erste Position einer solchen Kontaktstelle entscheidend. Diese Position, die auf Grund der Auslautverhärtung stets stimmlos ist (außer bei Nasalen und Liquiden), wirkt progressiv und verursacht bei einer nachfolgenden Lenis einen Stimmtonverlust. Die gesamte Konsonantenverbindung ist dann stimmlos zu sprechen. Dabei darf Stimmtonverlust nicht mit dem Verlust des Lenis-Charakters gleichgesetzt werden. Das [z] in ['apziçt] und das [d] in [kauf dire] bleiben Lenes, es sind aber im Gegensatz $\mathrm{zu}$ [z] und [d] keine stimmhaften Lenes, sondern stimmlose Lenes. Die in den Beispielen der Tabelle sichtbaren Assimilationsphänomene des Deutschen können in folgender Regel formuliert werden:

37 Die Transkription der deutschen und polnischen Beispiele erfolgt gemäß IPA, bei den deutschen Beispielen ist das Duden-Aussprachewörterbuch die Grundlage, unter Hinzuziehung des diakritischen Zeichens zur Markierung des Stimmtonverlusts. 
GWDA vs. Duden-Aussprachewörterbuch

Stimmhafte Lenis des Initialkonsonanten wird dann gefordert, wenn das Finalsegment ein Stimmhaftigkeit signalisierendes Graphem ist. Als stimmlose Lenis ist der Initialkonsonant dann zu artikulieren, wenn ihm ein Stimmlosigkeit signalisierendes Graphem oder ein auslautverhärtetes Segment vorausgeht. (RAUSCH / RAUSCH 1998:114) ${ }^{38}$

Beide untersuchten Aussprachewörterbücher des Deutschen weisen jeweils in der Einleitung korrekt auf das Problem des Stimmtonverlusts an der Wort- und Silbengrenze hin (vgl. Duden 2005:55, GWDA 1982:49, 72). Im Unterschied zur Einleitung, in der der Duden Wörter wie Absicht, Abwurf, abdanken als ['apžıcçt 'apyourf 'abdajkṇ] transkribiert, wird im Wörterverzeichnis ohne Angabe von Gründen auf das diakritische Zeichen zur Markierung des Stimmtonverlustes verzichtet, und dieselben Wörter erscheinen als ['apzıçt 'apvurf 'apdaykn]. Im GWDA wird auch im Wörterverzeichnis der Stimmtonverlust markiert, vgl. Schicksal ['Sıkzo:l], Absicht ['apzııçt], Abgang ['apg̊an]. Allerdings wird die Kennzeichnung inkonsequent gehandhabt, vgl. die GWDA-Transkriptionen von Wörtern wie Abwehr ['apve: ${ }^{\mathrm{p}}$ ], auswärts ['aosverts], obwohl [op'vo:l], in denen das diakritische Zeichen für den Stimmtonverlust fehlt. Ein Verzicht auf die Markierung des Stimmtonverlusts im Aussprachewörterbuch kann natürlich damit begründet werden, dass die Transkription trotz aller angestrebten Präzision auch für Laien lesbar bleiben muss, was durch ein Übermaß an diakritischen Zeichen erschwert wird. Und deutsche Muttersprachler, die das Aussprachewörterbuch als Nachschlagewerk in Zweifelsfällen benutzen, benötigen keinen zusätzlichen Hinweis auf den Stimmtonverlust, da sie die dem Phänomen zugrundeliegenden Regularitäten automatisch richtig verwenden. Ein Ausprachewörterbuch jedoch, das sich auch als Nachschlagewerk für Deutsch lernende Ausländer versteht, sollte gerade im Bereich des Stimmtonverlustes an der Wort- und Silbengrenze größtmögliche Detailliertheit anstreben. Die für das Polnische beschriebenen Abweichungen vom deutschen Assimilationsmuster treffen in dieser oder leicht abgewandelter Form auch für die meisten anderen europäischen Sprachen zu. Übungen zur Auslautverhärtung und zur progressiven Assimilation müssen im DaF-Unterricht nicht nur für Polen, sondern auch für Russen, Ukrainer, Slowaken, Tschechen, für Franzosen, Italiener, Spanier, Rumänen, für Ungarn und viele andere einen ihnen gebührenden Platz einnehmen. Didaktisch unterstützt werden könnten Fehlervermeidung und -korrektur in diesem Bereich zweifellos durch eine

$38 \mathrm{Zu}$ den Begriffen ,Stimmhaftigkeit / Stimmlosigkeit signalisierende Grapheme siehe RAUSCH / RAUSCH (1998:111). Ausführlich zu den Regularitäten der Auslautverhärtung und Assimilation vgl. RAUSCH / RAUSCH (1998:111-116, 234-244). 


\section{Gero Lietz}

Transkription, die dieses für das Deutsche so typische Phänomen systematisch und detailliert erfasst.

\section{Ausblick}

Ziel dieses Beitrages war es, eine kritische Bestandsaufnahme der Kodifikation der deutschen Standardaussprache vorzunehmen und den Nutzen einer korrekten und zugleich didaktisch begründeten Transkription für den DaF-Unterricht insbesondere in der Auslandsgermanistik zu diskutieren. Obwohl sowohl das GWDA als auch der Duden die IPA-Transkription verwenden, gibt es eine Reihe von kleineren und größeren Unterschieden, die im Zusammenhang mit der Phonetikausbildung der Germanistikstudierenden immer wieder für Verwirrung sorgen. Durch die systematische und detaillierte Gegenüberstellung dieser Diskrepanzen wird deutlich, dass es in der Phonetik - wie in anderen Bereichen der Sprachwissenschaft auch - natürlich in Einzelfragen verschiedene Modelle und Interpretationen der sprachlichen Wirklichkeit (in diesem Falle der Sprechrealität) gibt und sicherlich auch weiterhin geben wird. Gleichwohl ist es legitim - und dieses Anliegen hatte der Beitrag nicht zuletzt auch - bestimmte Konventionen in der IPA-Transkription des Deutschen, sei es nun im GWDA oder im Duden - zu hinterfragen mit dem Ziel, insbesondere für die Auslandsgermanistik ein bestmögliches Hilfsmittel und Nachschlagewerk zur Vermittlung der deutschen Standardaussprache zu erhalten. Dass dabei bei bestimmten Problemfeldern Lösungen des Duden favorisiert werden, in anderen Fragen Lösungen des GWDA, liegt in der Natur der Sache. Manche Bereiche, wie am Beispiel der Diphthonge gezeigt, verlangen möglicherweise die Realisierung von ganz neuen Ansätzen, die zwar angedacht sind, aber bislang weder in den Wörterverzeichnissen des GWDA noch des Duden ihren Niederschlag gefunden haben.

Der Anlass für diesen Beitrag ist ein sehr aktueller. Für Juni 2009 ist im Verlag Walter de Gruyter das Erscheinen des Deutschen Aussprachewörterbuches angekündigt, das auf ca. 1.000 Seiten ca. 150.000 Lemmata enthalten wird. ${ }^{39}$ In der Verlagsankündigung heißt es u. a.:

39 Vgl. die bibliographischen Angaben unter http://www.degruyter.com/cont/fb/sp/ detail.cfm?id=IS-9783110182026-1 (30.03.2008): Deutsches Aussprachewörterbuch. Von ANDERS, Lutz Christian / KRECH, Eva-Maria / STOCK, EBERHARD / Hirschfeld, Ursula. Mit Beiträgen von Wiesinger, Peter / HaAs, Walter / HOVE, INGRID. Erscheinungstermin: Juni 2009. ISBN 978-3-11-018202-6. 
GWDA vs. Duden-Aussprachewörterbuch

Das Deutsche Aussprachewörterbuch wurde von einer Gruppe renommierter Forscher des traditionsreichen Instituts für Sprechwissenschaft und Phonetik der Martin-Luther-Universität Halle-Wittenberg erarbeitet. Es ist - in der Nachfolge des halleschen Wörterbuchs der deutschen Aussprache, das bis 1982 in fünf Auflagen erschienen ist - das neue maßgebliche Referenzwerk zur deutschen Standardaussprache. Das Deutsche Aussprachewörterbuch informiert zuverlässig über den Aussprachestandard und ist normsetzend in allen Zweifelsfällen. ${ }^{40}$

Man darf gespannt sein, welche Lösungen das Deutsche Aussprachewörterbuch im Bereich der Transkription präsentiert. Einzelne Änderungen gegenüber der jetzigen Situation, das zeigen die obigen Ausführungen, scheinen unumgänglich zu sein. Vielleicht führen diese ja zu einer insgesamt größeren Einheitlichkeit der Kodifizierung der deutschen Standardaussprache, was sicherlich im Interesse der Phonetikausbildung für ausländische Germanistikstudierende läge. Der vorliegende Beitrag sei daher auch nicht zuletzt verstanden als Hilfe und Orientierung für Studierende und Lehrende, die möglicherweise bereits in einem Jahr mit dem neuen Aussprachewörterbuch arbeiten werden und sich natürlich die Frage nach den Vor- und Nachteilen verschiedener Ansätze und Lösungen in der IPA-Transkription stellen.

$40 \quad$ Vgl. http://www.degruyter.com/cont/fb/sp/detail.cfm?id=IS-9783110182026-1 (30.03. 2008). Allgemeine Informationen $\mathrm{zu}$ dem Werk sind auch dem Beitrag von HIRSCHFELD / STOCK (2007:13-15) zu entnehmen. Als Neuerungen hervorgehoben werden dort u.a. die geplanten umfangreichen Kapitel zur Eindeutschung von Wörtern und Namen aus fremden Sprachen sowie zur Standardaussprache in Österreich und in der deutschsprachigen Schweiz. Zu Neuerungen in der Transkription enthält der Beitrag keine konkreten Informationen. 
Gero Lietz

\section{Anhang}

Wichtige Unterschiede in der IPA-Transkription: Duden (2005), GWDA (1982)

\begin{tabular}{|c|c|c|}
\hline PHÄNOMEN & Duden & GWDA \\
\hline Qualität der $a$-Laute & $\begin{array}{l}\text { nur eine Qualität: } \\
\text { [a] [a:] } \\
\text { alle Aale: [alə '|a:lə] }\end{array}$ & $\begin{array}{l}\text { zwei Qualitäten: } \\
\text { [a] [a:] } \\
\text { alle Aale: [alə |'a:lə] }\end{array}$ \\
\hline Diphthonge & $\begin{array}{l}\text { [ai] [oy] [au] } \\
\text { mein neues Haus: } \\
\text { [main noyəs haus] }\end{array}$ & $\begin{array}{l}\text { [ae] [əø] [aо] } \\
\text { mein neues Haus: } \\
\text { [maen nọøəs haos] }\end{array}$ \\
\hline halblange Vokale & $\begin{array}{l}\text { Halblange Vokale } \\
\text { gibt es im Duden } \\
\text { nicht. } \\
\text { ['ki:no] } \\
\text { ['hari] } \\
\text { ['Ema] } \\
\text { ['e:mu] }\end{array}$ & $\begin{array}{l}\text { Halblange Vokale bei } \\
\text { volltonigem, unbetontem } \\
\text { Vokal im Auslaut. } \\
\text { ['ki:no'] Kino } \\
\text { ['hari'] Harry } \\
\text { ['Ema'] Emma } \\
\text { ['e:mu'] Emи }\end{array}$ \\
\hline $\begin{array}{l}\text { vokalisches } r \text { nach langem } \\
\text { Vokal innerhalb derselben } \\
\text { Silbe }\end{array}$ & $\begin{array}{l}\text { [bi:ph] } \\
\text { [e:e ên] } \\
\text { ['Umgəke:b्nt] }\end{array}$ & 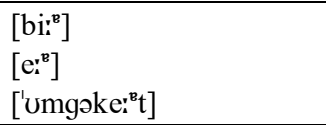 \\
\hline $\begin{array}{l}\text { vokalisches/konsonantisches } r \\
\text { nach langem } a \text {-Laut innerhalb } \\
\text { derselben Silbe }\end{array}$ & $\begin{array}{l}\text { [ha:e }] \\
{[\text { za:e }]}\end{array}$ & $\begin{array}{l}\text { [ha:r] } \\
\text { [za:r] }\end{array}$ \\
\hline $\begin{array}{l}\text { unbetonte Präfixe } e r-, \text { ver-, } \\
z e r-, \text { her- }\end{array}$ & 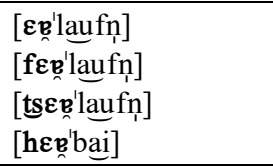 & $\begin{array}{l}\text { [e'laofn] } \\
\text { [fe'laofn] } \\
\text { [tse'laofn] } \\
\text { [he'bae ] }\end{array}$ \\
\hline $\begin{array}{l}\text { Reduzierung der Endung -en } \\
\text { nach Verschlusslauten } \\
\text { - nach }[\mathrm{b}],[\mathrm{p}] \\
\text { - nach }[\mathrm{g}],[\mathrm{k}] \\
\text { - nach }[\mathrm{d}],[\mathrm{t}]\end{array}$ & $\begin{array}{l}\text { ['lo:bṇ] } \\
\text { ['tro:pn] }] \\
\text { ['ly:gn] }] \\
\text { ['drykn] } \\
\text { ['fa:dn] } \\
\text { ['tre:tn] }\end{array}$ & $\begin{array}{l}\text { ['lo:bm] od. ['lo:bən] } \\
\text { ['tro:pm] od. ['tro:pən] } \\
\text { ['ly:gy] od. ['ly:gən] } \\
\text { ['drykn] od. ['drykən] } \\
\text { ['fa:dn] od. ['fa:dən] } \\
\text { ['tre:tn] od. ['tre:tən] }\end{array}$ \\
\hline
\end{tabular}


GWDA vs. Duden-Aussprachewörterbuch

\begin{tabular}{|c|c|c|}
\hline PHÄNOMEN & Duden & GWDA \\
\hline Endsilbenverbindung -igen & $\begin{array}{l}\text { reduziert: } \\
\text { [bə'zaitıgn }] \\
\text { [bə'rıçtıgn] }\end{array}$ & $\begin{array}{l}\text { volle Realisierung: } \\
\text { [bə'zaetigən] } \\
\text { [bə'rçtıgən] }\end{array}$ \\
\hline $\begin{array}{l}\text { schwachtoniges } e \text { nach }[\mathrm{g}] \text { in } \\
\text { der Endung - } e l\end{array}$ & $\begin{array}{l}\text { reduziert: } \\
\text { ['ri:gl] } \\
\text { ['re:gln] }\end{array}$ & $\begin{array}{l}\text { volle Realisierung: } \\
\text { ['ri:gəl] } \\
\text { ['re:gəln] }\end{array}$ \\
\hline $\begin{array}{l}\text { Affrikaten / } \\
\text { Explosiv-Frikativ-Verbindun- } \\
\text { gen }\end{array}$ & 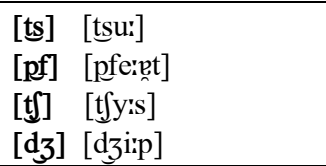 & $\begin{array}{l}\text { [ts] }[\text { tsu: }] \\
{[\mathrm{pf}] \quad\left[\mathrm{pfe:}{ }^{\mathrm{r}} \mathrm{t}\right]} \\
{\left[\mathrm{t} \int\right]\left[\mathrm{t} \int \mathrm{y}: \mathrm{s}\right]} \\
{[\mathrm{d} 3][\mathrm{d} 3 \mathrm{i}: \mathrm{p}]}\end{array}$ \\
\hline $\begin{array}{l}\text { Position des Symbols [l] für } \\
\text { den Stimmritzenverschlusslaut }\end{array}$ & 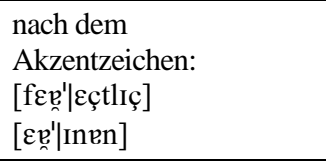 & $\begin{array}{l}\text { vor dem Akzentzeichen: } \\
\text { [fe|'Eçtlıç] } \\
\text { [e|'Inen] }\end{array}$ \\
\hline $\begin{array}{l}\text { Stimmritzenverschlusslaut bei } \\
\text { betonten Silben im Wortinnern } \\
\text { von Fremdwörtern }\end{array}$ & $\begin{array}{l}\text { ohne } \\
\text { Stimmritzenverschluss: } \\
\text { [te'a:te] } \\
\text { [muze'ail] } \\
\text { [negro'iit] } \\
\text { [ate'Ismus] }\end{array}$ & $\begin{array}{l}\text { mit } \\
\text { Stimmritzenverschluss: } \\
\text { [te|'a:te] } \\
\text { [muze|'a:l] } \\
\text { [negro|'itt] } \\
\text { [ate|'ısmus] }\end{array}$ \\
\hline $\begin{array}{l}\text { Kennzeichnung des } \\
\text { Stimmtonverlusts bei der } \\
\text { progressiven Assimilation }\end{array}$ & $\begin{array}{l}\text { Auf das Phänomen wird } \\
\text { in der Einleitung } \\
\text { hingewiesen. } \\
\text { Im Wörterverzeichnis } \\
\text { erfolgt jedoch keine } \\
\text { Kennzeichnung. }\end{array}$ & $\begin{array}{l}\text { Kennzeichnung auch im } \\
\text { Wörterverzeichnis, vgl.: } \\
\text { ['Jikza:l], ['apzıçt], } \\
\text { ['apğaj] } \\
\text { aber nicht konsequent, } \\
\text { vgl. u.a. ['apve:'], } \\
\text { ['aosverts] }\end{array}$ \\
\hline
\end{tabular}

\section{Literatur}

Boor, Helmut De / Diels, PAul (eds.) $\left({ }^{17} 1958\right)$ : Siebs. Deutsche Hochsprache. Bühnenaussprache. Berlin.

Boor, Helmut de / Moser, Hugo / Winkler, Christian (eds.) ( $\left.{ }^{19} 2000\right)$ : Siebs. Deutsche Aussprache. Reine und gemäßigte Hochlautung mit Aussprachewörterbuch. Nachdruck der 19. Auflage. Berlin.

Bussmann, HAdumOD (ed.) (2002): Lexikon der Sprachwissenschaft. Stuttgart.

CRUTtEndEn, AlAn (ed.) ( $\left.{ }^{5} 1994\right)$ : Gimson's Pronunciation of English. London. 
Gero Lietz

Dieling, Helga (1992): Phonetik im Fremdsprachenunterricht Deutsch. Berlin/ München/Wien u. a.

Dieling, Helga / Hirschfeld, Ursula (2000): Phonetik lehren und lernen. Fernstudieneinheit 21. München.

DomiśCZAK, HenRYK (1993): Wymowa w nauce języka niemieckiego. Koncepcje, metody, ćwiczenia. [Aussprache im Deutschunterricht. Konzeptionen, Methoden, Übungen]. Warszawa.

Duden (1962): Duden. Aussprachewörterbuch. Bearbeitet von Max Mangold in $\mathrm{Zu}-$ sammenarbeit mit der Dudenredaktion. Mannheim.

- (1974): Duden. Aussprachewörterbuch. Wörterbuch der deutschen Standardaussprache. 2., überarbeitete und aktualisierte Auflage. Bearbeitet von Max Mangold in $\mathrm{Zu}-$ sammenarbeit mit der Dudenredaktion. Mannheim.

- (1990): Duden. Aussprachewörterbuch. Wörterbuch der deutschen Standardaussprache. 3., völlig neu bearbeitete und erweiterte Auflage. Bearbeitet von Max Mangold in Zusammenarbeit mit der Dudenredaktion. Mannheim.

- (2000): Duden. Aussprachewörterbuch. Wörterbuch der deutschen Standardaussprache. 4., neu bearbeitete und aktualisierte Auflage. Bearbeitet von Max Mangold in $\mathrm{Zu}$ sammenarbeit mit der Dudenredaktion. Mannheim.

- (2005). Duden. Aussprachewörterbuch. 6., überarbeitete und aktualisierte Auflage. Bearbeitet von Max Mangold in Zusammenarbeit mit der Dudenredaktion. Mannheim.

EISENBERg, Peter ( $\left.{ }^{6} 1998\right)$ : Der Laut und die Lautstruktur des Wortes. In: DudenREDAKTION (ed.): Duden. Grammatik der deutschen Gegenwartssprache. Mannheim, 17-53.

GWDA (1982) = Großes Wörterbuch der deutschen Aussprache. Leipzig.

Hakkarainen, HeikKi J. (1995): Phonetik des Deutschen. München.

HERRMANN-WINTER, RENATE (1999): Neues hochdeutsch-plattdeutsches Wörterbuch. Rostock

HirschFeld, Ursula / ReinKe, Kerstin / Stock, Eberhard (2007): Phonothek intensiv. Aussprachetraining. Berlin/München.

HiRSCHFELD, URSUla / STOCK, EBERHARD (2007): Aussprachewörterbuch und DaFUnterricht. In: Zeitschrift für Interkulturellen Fremdsprachenunterricht [Online] 12:2. Abrufbar unter http://www.spz.tu-darmstadt.de/projekt_ejournal/jg-12-2/allgemein/ beitra33.htm

Høyem, Sturla / ZickfeldT, August Wilhelm (1990 / 21992): Deutsche Lautlehre. Oslo.

KeHR, RALF (2002): Versuch's auf Polnisch! Sprachführer für den deutsch-polnischen Jugendaustausch. Potsdam/Warszawa.

KoHler, Klaus J. (1977 / 21995): Einführung in die Phonetik des Deutschen. Berlin (=Grundlagen der Germanistik 20). 
GWDA vs. Duden-Aussprachewörterbuch

- (1999): German. In: Handbook of the International Phonetic Association. A guide to the use of the International Phonetic Alphabet. Cambridge, 86-89.

LANGHOFF, BARBARA (1996): A-Laute. In: STOCK / HiRSCHFELD, 52-56.

Lietz, Gero (2007): Rezension zu: BunK, Gerhard S. J. (2005): Phonetik aktuell. Kopiervorlagen mit 2 CDs. Ismaning. In: Zeitschrift für Interkulturellen Fremdsprachenunterricht [Online] 12:1. Abrufbar unter http://zif.spz.tu-darmstadt.de/jg-12-1/beitrag/ Bunk1.htm.

LOTZMANN, GEERT (1967): Zur Norm und Realisation der deutschen Hochlautung. In: Wirkendes Wort 1:228-238.

Madelska, Liliana / Witaszek-SAmborska, Mągorzata (1997 / $\left.{ }^{4} 1998\right)$ : Zapis fonetyczny. Zbiór ćwiczeń. [Phonetische Transkription. Übungssammlung]. Poznań.

MarcoPolo (1993 / 32005): Polnisch. Sprachführer mit Insider-Tipps. Stuttgart.

MEINHOLD, GOTTFRIED (1973): Deutsche Standardaussprache. Lautschwächungen und Formstufen. Jena (=Wissenschaftliche Beiträge der Friedrich-Schiller-Universität Jena).

Meinhold, GotTFried / StOck, Eberhard (1980 / 21982): Phonologie der deutschen Gegenwartssprache. Leipzig.

MiкоŁAJCZYк, Beata (2005): Deutsche Grammatik. Eine Einführung. Teil 1: Grundbegriffe. Phonetik und Phonologie. Poznań.

Morciniec, Norbert (1990): Das Lautsystem des Deutschen und des Polnischen. Heidelberg (=Deutsch im Kontrast 10).

Morciniec, Norbert / Prędota, StanisŁaw (1982 / 32005): Podręcznik wymowy niemieckiej. [Lehrbuch der deutschen Aussprache]. Warszawa.

Ramers, Karl Heinz (2001): Einführung in die Phonologie. München.

RAMERs, KARl HeInz / VATER, HeINZ (1988 / $\left.{ }^{2} 1991\right)$ : Einführung in die Phonologie. Hürth-Efferen (=Kölner Linguistische Arbeiten - Germanistik 16).

Rausch, Rudolf / Rausch, Ilka (1988 / ${ }^{5}$ 1998): Deutsche Phonetik für Ausländer. Leipzig.

Roach, Peter / Hartman, James / Setter, Jane (eds.) (1917 / ${ }^{17} 2006$ ): Daniel Jones. Cambridge English Pronouncing Dictionary. Cambridge.

SiEBS, THEODOR (1898): Deutsche Bühnenaussprache. Ergebnisse der Beratungen zur ausgleichenden Regelung der deutschen Bühnenaussprache, die vom 14. bis 16. April 1898 im Apollosaale des Königlichen Schauspielhauses zu Berlin stattgefunden haben. Berlin.

Stock, Eberhard / Hirschfeld, Ursula (eds.) (1996): Phonothek. Deutsch als Fremdsprache. Arbeitsbuch. Leipzig/Berlin/München.

TERnEs, Elmar (1987 / 21999): Einführung in die Phonologie. Darmstadt.

- (2002): Die phonetischen Angaben im de Gruyter Wörterbuch Deutsch als Fremdsprache. In: WIEGAND, HERBERT ERNST (ed.): Perspektiven der pädagogischen Lexikographie des Deutschen II. Tübingen, 125-135. 


\section{Gero Lietz}

VATER, HeInZ (2004): Wie viele Diphthonge hat das Deutsche? In: Estudios Filológicos Alemanes 4:7-21.

- (2005): Neue Phonologie-Theorien und ihre Anwendung aufs Deutsche. In: Kwartalnik Neofilologiczny 1:23-50.

WÄNGLER, HANS-HeINRICH (1960 / $\left.{ }^{4} 1983\right)$ : Grundriß einer Phonetik des Deutschen mit einer allgemeinen Einführung in die Phonetik. Marburg.

WDA (1964) = Wörterbuch der deutschen Aussprache . Leipzig.

WERNER-ULRICH, DORIS $\left({ }^{10} 2006\right)$ : Englisch Wort für Wort. Bielefeld. 